\title{
Orbital and physical parameters of eclipsing binaries from the ASAS catalogue - XII. A sample of systems with $K 2$ photometry
}

\author{
K. G. Hełminiak, ${ }^{1 \star}$ A. Moharana, ${ }^{1}$ T. Pawar, ${ }^{1}$ N. Ukita, ${ }^{2,3}$ P. Sybilski, ${ }^{1}$ \\ N. Espinoza, ${ }^{4}$ E. Kambe, ${ }^{5}$ M. Ratajczak, ${ }^{6}$ A. Jordán, ${ }^{7,8}$ H. Maehara, ${ }^{2}$ \\ R. Brahm, ${ }^{7,8}$ S. K. Kozłowski, ${ }^{9}$ and M. Konacki. ${ }^{9}$ \\ ${ }^{1}$ Nicolaus Copernicus Astronomical Center, Polish Academy of Sciences, ul. Rabiańska 8, 87-100 Toruń, Poland \\ ${ }^{2}$ Okayama Astrophysical Observatory, National Astronomical Observatory of Japan, 3037-5 Honjo, Kamogata, Asakuchi, \\ Okayama 719-0232, Japan \\ ${ }^{3}$ The Graduate University for Advanced Studies, 2-21-1 Osawa, Mitaka, Tokyo 181-8588, Japan \\ ${ }^{4}$ Space Telescope Science Institute, 3700 San Martin Dr., Baltimore 21218, MD, USA \\ ${ }^{5}$ Subaru Telescope, National Astronomical Observatory of Japan, 650 North Aohoku Place, Hilo, HI 96720, USA \\ ${ }^{6}$ Warsaw University Astronomical Observatory, Al. Ujazdowskie 4, 00-478 Warszawa, Poland \\ ${ }^{7}$ Facultad de Ingeniería y Ciencias, Universidad Adolfo Ibáñez, Av. Diagonal las Torres 2640, Peñalolén, Santiago, Chile \\ ${ }^{8}$ Millennium Institute for Astrophysics, Santiago, Chile \\ ${ }^{9}$ Institute of Automatic Control and Robotics, Poznań University of Technology, Piotrowo 3A, 61-131 Poznań, Poland \\ ${ }^{10}$ Nicolaus Copernicus Astronomical Center, Polish Academy of Sciences, ul. Bartycka 18, 00-716 Warszawa, Poland
}

Accepted XXX. Received YYY; in original form ZZZ

\begin{abstract}
We present results of the analysis of light and radial velocity (RV) curves of eight detached eclipsing binaries observed by the All-Sky Automated Survey, which we have followed up with high-resolution spectroscopy, and were later observed by the Kepler satellite as part of the K2 mission. The RV measurements came from spectra obtained with OAO-188/HIDES, MPG2.2m/FEROS, SMARTS 1.5m/CHIRON, Euler/CORALIE, ESO-3.6m/HARPS, and OHP1.93/ELODIE instruments. The $K 2$ time-series photometry was analysed with the JKTEBOP code, with out-of-eclipse modulations of different origin taken into account. Individual component spectra were retrieved with the FD3 code, and analysed with the code ISPEC in order to determine effective temperatures and metallicities. Absolute values of masses, radii, and other stellar parameters are calculated, as well as ages, found through isochrone fitting. For five systems such analysis has been done for the first time. The presented sample consists of a variety of stars, from low-mass dwarfs, through G and F-type Main Sequence objects, to evolved active sub-giants, one of which is found to be crossing the Hertzsprung gap. One target may contain a $\gamma$ Dor-type pulsator, two more are parts of higher-order multiples, and spectra of their tertiaries were also retrieved and used to constrain the properties of these systems.
\end{abstract}

Key words: binaries: eclipsing - binaries: spectroscopic - stars: fundamental parameters stars: late-type - stars: individual: HD 284753, EPIC 202073040, RU Cnc, BD+18 2050, FM Leo, HD 149946, BD-19 4582, HD 219869

\section{INTRODUCTION}

Double-lined detached eclipsing binaries (DEBs) are among the most useful objects in astrophysics. Their photometric and spectroscopic observations allow one to determine basic stellar parameters and carry out a wide range of tests of stellar structure, evolution and dynamics. They play important role for such branches of astronomy as galaxy structure and evolution, stellar populations, formation and evolution of exoplanets or tidal interactions. In order to be consid- ered useful, a DEB needs to have parameters of its components (i.e. mass and radius) derived with the accuracy of $<3 \%$ (Clausen et al. 2008; Lastennet \& Valls-Gabaud 2002), and even down to $\sim 0.1 \%$ to challenge the modern models of stellar evolution (Valle et al. 2017). A surprisingly low number of DEBs have their absolute masses and radii derived accurately enough. In an up-to-date DEBCat catalog (Southworth 2015) ${ }^{1}$, there are only 271 systems listed (at the moment of writing this article). In a groundbreaking review, Torres et al. (2010) point out the lack of well-characterized stars 
at the high- and low-mass end of the main sequence, or on very early or late stages of evolution. In the same time they notice that evolutionary models often fail to reproduce the observed values of parameters for a variety of objects, due to improper treatment of the convection, overshooting or magnetic fields for instance. Future models will attempt to fix these discrepancies, but they can only be verified with a sufficient number of high-quality data. Such data can only come from observations of many objects of a particular class. Meanwhile, except for F, G and early K-type main sequence (0.8$2.0 \mathrm{M}_{\odot}$ ) stars, we see a lack of sufficient number of well-studied objects.

As a response to Torres' call, in 2011 we started a large spectroscopic programme, aimed for an overall characterization of several hundred DEBs, including numerous new cases of stars occupying the under-populated regions in both southern (see other papers of this series, e.g.: Hełminiak et al. 2014; Hełminiak et al. 2015; Hełminiak et al. 2019a; Ratajczak et al. 2013; Coronado et al. 2015) and northern hemispheres (e.g. Hełminiak et al. 2019b). This survey provided thousands of high-resolution spectra and precise radial velocity $(\mathrm{RV})$ measurements, which need to be supplemented with high-precision photometry. The most precise are from spacebased observatories, such as Kepler, or TESS. We exploited the Kepler main field (Hełminiak et al. 2016; Hełminiak et al. 2019b), characterizing a majority of bright $(V<12 \mathrm{mag})$ and not previously studied DEBs, and the ongoing TESS observations are already providing data for many of our targets (Hełminiak et al. 2019c; Marcadon et al. 2020; Ratajczak et al. 2021). In this work we focus on those systems from our survey, that fell into the field of view of the Kepler satellite during its $K 2$ mission.

The paper is organized as follows. In Section 2 we present our sample of DEBs; in Sect. 3 we present the spectroscopic and photometric data sets; Sect. 4 describes the methodology of data analysis used in this work; Sect. 5 presents the results, including estimates of ages; and finally Sect. 6 concludes our findings.

\section{TARGETS}

All presented DEBs were observed and/or discovered by the All-Sky Automated Survey (ASAS; Pojmański 2002), including its northern counterpart (ASAS-N). With one exception, they are listed in the ASAS Catalog of Variable Stars (ACVS). They were included into target list of the previously mentioned, large spectroscopic survey, which we conducted in years 2011-2018 on a number of telescopes and spectrographs, and which focused on relatively bright $(V<12 \mathrm{mag}$ ) detached binaries of spectral types $\mathrm{F}$ to $\mathrm{M}$ (observed colour $V-K>1.1 \mathrm{mag}$ ). Spectroscopic observations were done independently from and started usually before the $K 2$ mission. Below we briefly describe each of our targets (ordered by increasing right ascension):

- ASAS J045021+2300.4 = EPIC 247605441, HD 284753, $B D+22760$ (hereafter: A-045): This system was first recognized as an eclipsing variable star from the ASAS-N photometry by Kiraga \& Stępień (2013), and is the only case from this work not listed in the ACVS. Nevertheless, it is close to the northern edge of the field of view of the southern ASAS station $\left(\delta<+28^{\circ}\right)$, so its pre-2009 photometry is also available. It has been associated with a ROSAT Bright Source Catalogue (Voges et al. 1999) object 1RXS 045021.0+230037, which suggests its strong activity. No detailed study of this system has been conducted so far.

- ASAS J060505+2032.1 = EPIC 202073040, TYC 1321-16-1 (hereafter: A-060): This system was discovered as a DEB by the
ASAS, but the period given in the ACVS $(P \simeq 33.371 \mathrm{~d}$ ) turned out to be incorrect. Later, it was identified in $K 2$ photometry first by Armstrong et al. (2015), and shortly after by LaCourse et al. (2015). The correct orbital period ( $P \simeq 2.121 \mathrm{~d}$ ) was found only in the latter work, while Armstrong et al. (2015) gave a value close to $P / 2$. No detailed study of this system has been conducted so far.

- ASAS J083730+2333.7 = EPIC 212173112, RU Cnc, BD+24 1959, HIP 42303 (hereafter: A-083): This star was discovered as a variable in 1908 by L. Ceraski, and designated at that time as 3.1911 Cancri (Ceraski 1911). It is first mentioned as an eclipsing binary by Shapley (1913). Numerous studies were published since then, including two with RV curves: Popper (1990), and Imbert (2002). Popper (1990) also performed a light curve analysis, and estimated absolute values of masses and radii. Majority of estimates of the effective temperature that are available in the literature, were done under the assumption of the target being a single star, therefore they are not reliable. Most recently, Çokluk et al. (2019) analysed the $K 2$ photometric data and the two historical RV curves. In this work, we calculated the RVs from our own, as well as unpublished archival spectra, and used a different approach to the light curve analysis than Çokluk et al. (2019).

- ASAS J085002+1752.5 = EPIC 211839462/-30, BD+18 $2050 A B, A D S 7030 A B$ (hereafter: A-085): This DEB, discovered as an eclipsing variable by ASAS, and independently identified by the KELT survey (Pepper et al. 2008), is a part of a visual pair ADS $7030 \mathrm{AB}$ (a.k.a. WDS 08500+1752AB, CCDM J08500+1752AB, KU 33), whose components differ in brightness by about 0.3 mag. The corresponding EPIC designations are 211839462 for the brighter, western star A, and 211839430 for the fainter component B to the East. The component B is currently incorrectly noted as a DEB in Simbad and in the Washington Double Star Catalogue (WDS; Mason et al. 2001), probably because of the original ASAS identification. However, our spectroscopic observations revealed a single-lined spectrum of the component $\mathrm{B}$, and a double-lined and rotationally broadened spectrum of component A, with RV changes clearly seen. This has been confirmed by the analysis of $K 2$ data, when deeper eclipses were identified on the position of EPIC...62 rather than on ...30 (Barros et al. 2016). Atmospheric parameters, such as effective temperature $T_{\text {eff }}$, logarithm of gravity $\log (g)$ or metallicity $[\mathrm{M} / \mathrm{H}]$ for the component B can be found in the literature (Huber et al. 2016; Ho et al. 2017; Ting et al. 2018; Gaia Collaboration 2018), all pointing to a red giant with $T_{\text {eff }} \simeq 5000 \mathrm{~K}$, but not very consistent in terms of other values. Lee (2015) used the ASAS light curve and the MECI (Devor et al. 2008) code to find the most probable masses and age of the eclipsing binary, but only got limits for these parameters.

- ASAS J111245+0020.9 = EPIC 201488365, FM Leo, HD 97422, BD+01 253, HIP 54766 (hereafter: A-111): This DEB was discovered by the Hipparcos mission, and found its place in the 74th Special Name-List of Variable Stars (Kazarovets et al. 1999). The first RV and light curve solutions were presented by Ratajczak et al. (2010), but the quality of the photometric data was far from optimal. More recently, Maxted \& Hutcheon (2018) combined the $K 2$ data with RVs from Ratajczak et al. (2010), and obtained very precise results. Another set of RV measurements, including large number of spectra taken during eclipses, was combined with the ASAS light curve and used to model the system by Sybilski et al. (2018), who also showed the spin-orbit alignment of the two components. Finally, Graczyk et al. (2021) used new HARPS spectra, together with the $K 2$ photometry, and presented a comprehensive analysis, including spectra decomposition, atmospheric parameters, and eclipse timing variations. In this work, we use our own RVs calculated from: 
Table 1. Basic information about the presented systems, as listed in Simbad. Positions, proper motions and distances come from the Gaia Early Data Release 3 (GEDR3) catalogue (Gaia Collaboration 2021). The $K$-band magnitudes are from 2MASS (Cutri et al. 2003).

\begin{tabular}{|c|c|c|c|c|c|c|c|c|c|}
\hline ASAS ID & EPIC ID & Other ID & $\begin{array}{l}\text { RA } \\
\left({ }^{\circ}\right)\end{array}$ & $\begin{array}{c}\text { DEC } \\
\left({ }^{\circ}\right)\end{array}$ & $\begin{array}{c}\mu_{\mathrm{RA}} \\
\left(\operatorname{mas~yr}^{-1}\right)\end{array}$ & $\begin{array}{c}\mu_{\mathrm{DEC}} \\
\left(\mathrm{mas} \mathrm{yr}^{-1}\right)\end{array}$ & $\begin{array}{c}V \\
(\mathrm{mag})\end{array}$ & $\begin{array}{c}K \\
(\mathrm{mag})\end{array}$ & $\begin{array}{c}d_{G E D R 3} \\
(\mathrm{pc})\end{array}$ \\
\hline $045021+2300.4$ & 247605441 & HD 284753 & 72.585989 & +23.005982 & $1.4(4)$ & $-40.9(3)$ & 10.26 & 8.176 & $100(3)$ \\
\hline $060505+2032.1$ & 202073040 & TYC 1321-16-1 & 91.270401 & +20.536897 & $1.64(2)$ & $-4.72(2)$ & 11.93 & 10.814 & $1095(25)$ \\
\hline $083730+2333.7$ & 212173112 & RU Cnc & 129.375422 & +23.561564 & $-21.62(2)$ & $-0.81(1)$ & 10.20 & 8.015 & 408(3) \\
\hline $085002+1752.5$ & 211839462 & $\mathrm{BD}+182050 \mathrm{~A}$ & 132.505874 & +17.874540 & $-9.06(2)$ & $-4.20(1)$ & 10.15 & 9.145 & $522(4)$ \\
\hline $111245+0020.9$ & 201488365 & FM Leo & 168.187464 & +0.347869 & $-96.73(3)$ & $-32.24(2)$ & 8.45 & 7.211 & $146.4(6)$ \\
\hline $163903-2847.2$ & 202674012 & HD 149946 & 249.764233 & -28.787116 & $15.49(2)$ & $-11.94(2)$ & 9.85 & 8.326 & $267.3(1.4)$ \\
\hline $171750-1915.3$ & 234440875 & BD-19 4582 & 259.456832 & -19.254559 & $-6.75(2)$ & $-10.98(1)$ & 10.99 & 9.178 & $368(2)$ \\
\hline $231922-0852.2$ & 246024234 & HD 219869 & 349.842294 & -8.870378 & $52.36(2)$ & $10.63(2)$ & 10.24 & 8.990 & $289.6(1.6)$ \\
\hline
\end{tabular}

(1) the out-of-eclipse spectra from Sybilski et al. $(2018)^{2}$, (2) the HARPS spectra used by Graczyk et al. (2021), and (3) publicly available FEROS spectra, not used in any publication so far. We also present our own approach to the $K 2$ data, independently from Maxted \& Hutcheon (2018) and Graczyk et al. (2021).

- ASAS J163903-2847.2 = EPIC 202674012, HD 149946 (hereafter: A-163): This system was first identified as a DEB by ASAS. Recently, the $K 2$ light curve was analysed by Maxted \& Hutcheon (2018) together with four publicly available FEROS spectra from our spectroscopic survey, leading to a preliminary solution for this object. Later, in a short Research Note (Hełminiak et al. 2018), we presented a more precise orbital solution from 15 high-resolution spectra, which we combined with LC-based parameters from Maxted \& Hutcheon (2018). Recently, Hoyman \& Çakırlı (2020) presented their study, in which they used CORALIE, CHIRON and one FEROS RV measurements from Hełminiak et al. (2018), their own measurements for three archival FEROS and three HARPS spectra, own light curve modelling, and atmospheric parameters obtained from disentangled component spectra. In this work, we repeat the orbital analysis with the addition of ten HARPS spectra from the ESO archive, and explain the process in more details. Furthermore we present our own approach to the $K 2$ data, not relying on results of Maxted \& Hutcheon (2018) nor Hoyman \& Çakırlı (2020).

- ASAS J171750-1915.3 = EPIC 234440875, BD-19 4582 (hereafter: A-171): Another DEB discovered by ASAS, although the orbital period given in the ACVS is slightly longer than the true value, which affected the phase-folded light curve. No detailed study of this system has been conducted so far.

- ASAS J231922-0852.2 = EPIC 246024234, HD 219869, BD096166 (hereafter: A-231): This star was first identified as a DEB in the ACVS, and later in data from the STEREO satellite (Wraight et al. 2011). In both cases, however, the given orbital period is close to half of the true value. Two spectroscopic observations were taken by the RAVE survey (Kordopatis et al. 2013; Kunder et al. 2017), and the measured RVs differ by over $50 \mathrm{~km} \mathrm{~s}^{-1}$, which indicates a spectroscopic binary. Apart from that, no other study of this system has been performed.

2 FM Leo is an exception in the sample, since after the work of Ratajczak et al. (2010) we did not intend to include it into our large spectroscopic survey. We changed this after P. Sybilski realized that this star was very well suited for studying the Rossiter-McLaughlin effect.

\section{DATA}

\subsection{Spectroscopic observations and data reduction}

Spectra of the presented systems come mainly from our own large spectroscopic survey, and were taken with four major instruments we used in the project.

The CHIRON spectrograph (Schwab et al. 2012; Tokovinin et al. 2013; Paredes et al. 2021), attached to the 1.5-m SMARTS telescope in the Cerro Tololo Inter-american Observatory (CTIO, Chile), was used in the "slicer" and "fiber" modes, which provide spectral resolution of $R \sim 90000$ and $\sim 28000$, respectively. The latter provides much higher efficiency. This telescope works in service mode only. Spectra were reduced with the pipeline developed at Yale University (Tokovinin et al. 2013). Wavelength calibration is based on ThAr lamp exposures taken just before the science observation. Barycentric corrections are not applied by the pipeline, thus we were calculating them ourselves under IRAF ${ }^{3}$ with bcvcor task. For the targets described here we did not use the available iodine $\left(\mathrm{I}_{2}\right)$ cell. Without the $\mathrm{I}_{2}$, the stability of the instrument is estimated to be better than $15 \mathrm{~m} \mathrm{~s}^{-1}$ in "slicer" mode. For the radial velocity (RV) measurements we used 36 echelle orders, spanning from 4580 to $6500 \AA$ (limited by the templates we used), but the complete spectrum reaches $8760 \AA$.

The observations at Okayama Astrophysical Observatory (OAO) 1.88-m telescope in Okayama (Japan) with the HIDES spectrograph (Izumiura 1999; Kambe et al. 2013) were conducted in the fibre mode with image slicer $(R \sim 50000)$, without $\mathrm{I}_{2}$, and with ThAr lamp frames taken every 1-2 hours. The spectra are composed of 62 rows covering 4080-7538 $\AA$, of which we use 30 (4365$6440 \AA$ ). Detailed description of the observing procedure, data reduction and calibrations is presented in Hełminiak et al. (2016). The precision reached with our approach is $40-50 \mathrm{~m} \mathrm{~s}^{-1}$.

The CORALIE spectrograph, attached to the 1.2-m Euler telescope in La Silla (Chile), works in a simultaneous object-calibration mode, and provides resolution of $R \sim 70000$. Additional ThAr exposures with both fibres are done every 1-1.5 hours. For this study we used the instrument when it was still equipped with circular fibres (currently octagonal). Spectra were reduced with the dedicated python-based pipeline (Jordán et al. 2014; Brahm et al. 2017), which also performs barycentric corrections. The pipeline is optimized to derive high-precision radial velocities, down to $\sim 5$

${ }^{3}$ IRAF is distributed by the National Optical Astronomy Observatory (NOAO), which is operated by the Association of Universities for Research in Astronomy (AURA) under cooperative agreement with the National Science Foundation. http://iraf.noao.edu/ 
$\mathrm{m} \mathrm{s}^{-1}$, and reduces the spectrum to 70 rows spanning from 3840 to $6900 \AA$. For our purposes, we use only 45 rows (4400-6500 $⿱$ ) ), due to the limits of our template spectra and very low signal in the blue part.

Operations at the MPG-2.2m telescope (La Silla, Chile) with the FEROS instrument (Kaufer et al. 1999) look very similar to CORALIE, as the spectrograph also works in a simultaneous objectcalibration manner, but employs an image slicer, which gives $R$ 48000 , and the highest efficiency of all the optical instruments we used for this study ( $>20 \%$ ), thus provides data with the highest SNR. Spectra were reduced with a similar pipeline as for CORALIE, capable of providing RVs with the precision of $5-8 \mathrm{~m} \mathrm{~s}^{-1}$. Although the original spectral format reaches beyond $10000 \AA$, the output is reduced to 21 rows covering 4115-6519 $\AA$, of which we use 20 (4135-6500 ̊̊).

A single spectrum of a visual companion to the A-085 system was obtained with the HDS (Noguchi et al. 2002) instrument attached to the 8.2-m Subaru telescope, located on Maunakea, Hawaii. The observation was taken through a slit $\sim 0.65$ wide, which resulted in $R \sim 55000$. The 720-second exposure resulted in SNR 103. A Th-Ar lamp was used for wavelength calibration. This spectrum was reduced and calibrated with dedicated IRAF procedures. It was only used to evaluate the metallicity of the A- 085 system.

Data from these instruments were supplemented with archival spectra from the HARPS spectrograph, attached to the ESO 3.6-m telescope in La Silla, the FEROS instrument behind the MPG$2.2 \mathrm{~m}$ telescope in La Silla, and ELODIE, which was the highresolution instrument of the 1.93-m telescope of the Observatoire de Haute-Provence (OHP). These data were extracted from the $\mathrm{ESO}^{4}$ and ELODIE $^{5}$ (Moultaka et al. 2004) archives, respectively. From FEROS and HARPS observations, we only used spectra reduced and calibrated with the local Data Reduction Pipeline (DRP). The HARPS data are available in two modes: high-resolution (ECHE; $R \sim 115000$ ) and high-efficiency (EGGS; $R \sim 80000$ ). In case of ELODIE, we retrieved single reconnected spectra, resampled in wavelength with a constant step of $0.05 \AA$ (the nominal spectral resolution of the instrument is $R \sim 42000$ ), covering the range 4000-6800 A. If archival spectra were used, their number does not exceed the number of our own observations, with the exception of A111 = FM Leo.

Below we summarise spectroscopic data sets for each target separately:

- A-045: The observations were done only with HIDES. They started in December 2014, and lasted till November 2017. A total of 22 spectra were taken that time. Because of the faintness of the secondary, in some cases measurement of its RV was not possible. We therefore have 22 and 17 data points for the primary and secondary, respectively.

- A-060: Observed only with CHIRON in the "fiber" mode, mainly in 2014, when eight spectra were recorded. Two additional visits were made in 2019 , in order to improve the coverage and verify the existence of a possible long-term RV trend.

- A-083: Observed by us only with HIDES. A total of 11 spectra were taken between December 2014 and December 2015. Five additional unpublished spectra, from March 1998, were found in the ELODIE archive.

- A-085: Observations started on HDS in October 2011, and on CORALIE in March 2012, but because of the confusion on which

\footnotetext{
${ }^{4}$ http://archive.eso.org/wdb/wdb/adp/phase3_main/form

5 http://atlas.obs-hp.fr/elodie/
}

component of the visual pair is the eclipsing binary, the wrong one was observed. Observations of the correct one started in November 2013, and lasted till May 2014. Four CORALIE and seven CHIRONfiber spectra were taken that time. One of the CHIRON spectra was taken during the total part of the primary eclipse, and was not used for RV calculations.

- A-111: A large number of CHIRON spectra were taken in the "slicer" mode during a dedicated campaign in February and March 2016 (Sybilski et al. 2018). From this set, 13 observations were taken outside of the eclipses, and used for disentangling and orbital fit. In this work, we use these disentangled spectra to derive atmospheric parameters. For RV calculations we use the original 13 out-of-eclipse observations, and, additionally, a set of HARPS (ECHE; 12) and FEROS (8) spectra of A-111, both available in the ESO archive. The HARPS data were used in Graczyk et al. (2021), but the FEROS spectra were not. We decided to use them both for RV calculations, but not for disentangling nor spectral analysis. Furthermore, for this target we did not use the RV measurements from Ratajczak et al. (2010), which are of lower precision. Their inclusion did not affect the final solution, but led to larger uncertainties.

- A-163: Five CHIRON spectra in the "slicer" mode were taken in 2012, alongside with two CORALIE and two FEROS spectra. Further CORALIE (three) and FEROS (one) visits were made in 2013, and later in 2015 (one for both instruments). These 13 spectra constitute the data used for the orbital solution presented in Hełminiak et al. (2018). Ten additional HARPS spectra (seven ECHE and three EGGS), taken in 2018 and 2019, were found in the ESO archive.

- A-171: This target was observed with FEROS six times in 2013, and once in 2015. Six additional CORALIE spectra were taken between July 2013 and June 2016. Finally, two HARPS spectra (ECHE), from May 2018 and April 2019, have been extracted from the ESO archive.

- A-231: We have observed this star with FEROS between June 2012 and June 2015, taking 12 spectra in total. Additionally, three HARPS spectra (EGGS), taken in December 2017, have been extracted from the ESO archive.

\subsection{RV measurements}

In our spectroscopic survey we measure radial velocities of components of a binary system using our own implementation of the TODCOR technique (Zucker \& Mazeh 1994). As templates we use synthetic spectra computed with ATLAS 9 (Kurucz 1992), which do not reach wavelengths longer than $6500 \AA$. This lowers the number of useful echelle orders, but reduces the influence of telluric lines, and cuts off the $\mathrm{H} \alpha$ line. We also do not take into account orders with the sodium D lines ( $5900 \AA)$, as they are often affected by the interstellar medium. Initially we use templates calculated for effective temperatures roughly expected from the spectral type of the binary, but for the final measurements and orbital fit, we select templates based on $T_{\text {eff }}$ expected for a given mass and radius, and rotationally broadened. Uncertainties are calculated with a bootstrap procedure (Hełminiak et al. 2012), which is sensitive to the SNR of a component, and velocity of rotation. The TODCOR procedure also provides the ratio of component fluxes, that maximizes the value of the CCF at the position of the resulting RVs. These flux ratios can be later used to rescale and renormalise disentangled spectra before their analysis. Their reliability was verified at several occasions with the aid of spectra taken during a total eclipse, e.g. in Hełminiak et al. (2015), Ratajczak et al. (2021), or here in the case of A-085. 
Table 2. Summary of $K 2$ data used in this study

\begin{tabular}{ccccl}
\hline \hline EPIC ID & Cam. & Adopted & No. of data & Notes \\
\hline 247605441 & 13 & EVEREST & 3939 & 5 parts \\
202073040 & 0 & EVEREST & 1528 & \\
212173112 & 5 & EVEREST & 3577 & 3 parts \\
, & 18 & EVEREST & 2329 & 2 parts \\
211839462 & 5 & EVEREST & 3596 & 3 parts \\
, & 16 & EVEREST & 3857 & 5 parts \\
$"$ & 18 & EVEREST & 2456 & 2 parts \\
201488365 & 1 & EVEREST & 3513 & 5 parts, SC \\
202674012 & 2 & EVEREST & 3247 & \\
234440875 & 11 & K2PDC & 745 & C11a only \\
246024234 & 12 & EVEREST & 3383 & 3 parts \\
$"$ & 19 & K2SFF & 339 & Single period \\
\hline
\end{tabular}

\section{3 $K 2$ photometric data}

The studied systems were observed by the Kepler space telescope during several campaigns of its extended $K 2$ mission (Howell et al. 2014). The photometric time series data were extracted from the Mikulski Archive for Space Telescopes (MAST) ${ }^{6}$. We usually took 30-minute long-cadence (LC) data, except for A-111, for which short-cadence (SC) data also exist. Also available in MAST are SC data for A-083, but we decided not to work on them, and the reasons behind this decision will be explained later in the text. The $K 2$ data are strongly affected by systematics coming from the pointing of the satellite, and its roll angle variations. Several automated algorithms have been developed in order to correct for these effects, such as: EVEREST (Luger et al. 2016, 2018), K2sFF (Vanderburg \& Johnson 2014), or POLAR (Barros et al. 2016). They produce light curves designated as High-Level Science Products (HLSP), available for download from the MAST as calibration level 4 data. Apart from them, the Archive contains flux measurements with lower calibration level 2, which include Simple Aperture Photometry (SAP) data, and measurements corrected with the Presearch Data Conditioning (PDC) method (Stumpe at al. 2012). In this paper we will refer to the latter as K2PDC.

Not all HLSP products are available for the studied systems. Also, each algorithm tackles short- and long-term systematic trends in different ways, therefore produces different light curves. In some occasions this can affect the intrinsic out-of-eclipse variations, or even depths and shapes of the eclipses. For these reasons we have collected all available data (calibration level 2 and 4), and have inspected them for each system and campaign individually. We mainly took into account the stability of the shape and depth of eclipses, as well as the out-of-eclipse variations and number of outliers. Sometimes modelling was done on two different products, and results of the one that produced lower parameter errors and $\mathrm{rms}$ of the fit were adopted.

Table 2 summarizes the $K 2$ data adopted for each system and campaign. Figure B1 in the Appendix shows all the adopted light curves, after cleaning, as a function of time. For clarity, the lower panels show zooms on the out-of-eclipse modulations (coming from cold spots and detrending procedures), which often evolve in very short time scales, of single orbital periods. In some cases, the detrending algorithm left a discontinuity in the light curve. In the case of A-085 (EPIC 211839462) the varying pointing of the telescope resulted in changes of the third light contamination. For all these reasons, the majority of light curves were analysed in parts. Their number per target and campaign, as well as the total number of data points in a given campaign, are given in Table 2. In Figure B1, different parts are represented by different colours.

Finally, we would like to note that for A-171 (EPIC 234440875) we only took data from the first part of Campaign 11. This campaign was separated into two segments as a result of an error in the initial roll-angle used to minimize solar torque on the spacecraft ${ }^{7}$. Data from the second part are of worse quality, with significantly larger scatter and short-term systematics, which led to larger uncertainties of the resulting parameters. The adopted data set continuously covers about five orbital periods, which we find enough for a proper analysis.

\section{ANALYSIS}

\subsection{RV fitting}

The RV solutions were found using the procedure called v2FIT (Konacki et al. 2010). We used it to fit a double-Keplerian orbit to a set of RV measurements of two components, utilizing the Levenberg-Marquardt minimization scheme. The fitted parameters are: orbital period $P$, zero-phase moment $T_{P}{ }^{8}$, systemic velocity $\gamma$, velocity semi-amplitudes $K_{1,2}$, eccentricity $e$ and periastron longitude $\omega$. Depending on the case, we also included the difference between systemic velocities of two components, $\gamma_{2}-\gamma_{1}$, and difference between zero points of different spectrographs. Whenever applicable, we simplified our fit by keeping the orbital period on the value given by initial fits to complete light curves (see next Section), or by fixing $e, \gamma_{2}-\gamma_{1}$, or instrument zero points differences to zero, when any of these parameters was found indifferent form 0.0.

Systematic errors that come from fixing a certain parameter in the fit are assessed by a Monte-Carlo procedure, which perturbs the value of such parameter within its given error (e.g. when orbital period is known from light curve analysis). Other possible systematics (like coming from poor sampling, low number of measurements, pulsations, activity etc.) are estimated by a bootstrap analysis. All the uncertainties of orbital parameters given in this work already include the systematics.

Moreover, to obtain reliable formal parameter errors of the fit, and the final reduced $\chi^{2}$ to be close to 1 , we were modifying the RV measurement errors either by adding a systematic term (jitter) in quadrature, or multiplying by a certain factor. Adding the jitter works better for active stars, like A-083 or A-231, when the RV scatter is caused by spots, and is compensated with the additional term. However, since v2FIT weights the measurements on the basis of their own errors, which are sensitive to SNR and rotational velocity, we mainly used the second option, in which the weights are preserved, and which is more suitable for stars with significant flux difference (like A-045).

\subsection{Light curve fitting}

The $K 2$ light curves were fitted with the version 40 (v40) of the code JктевоP (Southworth et al. 2004a,b), which is based on the EBOP program (Popper \& Etzel 1981). It is designed to work with well-separated binaries, with the "oblateness" of components not

7 More details in https: //keplerscience.arc.nasa.gov/k2-data-release-note

8 Defined in this code as the moment of passing the pericentre for eccentric

${ }^{6}$ https://mast.stsci.edu/portal/Mashup/Clients/Mast/Portal.htmlorbits or quadrature for circular. 
exceeding $4 \%$. The highest value in our sample was found to be $1.1 \%$ (A-060), thus the use of Јктевор is justified.

The code fits the period $P$, mid-time of the primary (deeper) minimum $T_{0}$, sum of the fractional radii $r_{1}+r_{2}$ (where $r=R / a$ ), their ratio $k$, inclination $i$, surface brightness ratio $J$, maximum brightness $S$, as well as for $e$ and $\omega$, however their final values are actually from v2FIT runs, unless stated otherwise. Third light contribution $l_{3} / l_{\text {tot }}$, which can be significant in $K 2$ data, was also initially fitted for, but when it was found indifferent from zero, the fit was repeated with fixed $l_{3} / l_{\text {tot }}=0$. It is worth noting that the detrending algorithms already correct for additional light from nearby sources (e.g. PDC), therefore the $l_{3}$ value does not always have a physical meaning, unless the SAP curve is being modelled. In some cases the contamination level can be overestimated, and in the fitting process one can even obtain a negative value of $l_{3}$.

The gravity darkening coefficients and bolometric albedos were always kept fixed at the values appropriate for stars with convective envelopes ( $g=0.32, A=0.5$; Lucy 1967; Rucinski 1969). Reflection coefficients were fixed at zero, with the exception of A-171, where they were treated as free parameters. For the limb darkening (LD) coefficients we used the logarithmic law of (Klinglesmith \& Sobieski 1970). Initial values were found on the basis of $\log (g)$ found from first v2FIT and JKTEBOP runs, and temperatures from approximate isochrones (of solar metallicity). In the final runs, the LD coefficients were fine-tuned during a fit, or at least perturbed during the error estimation stage. In some cases, setting the LD free led to physically impossible values, which might be explained for example by spots affecting the shape of an eclipse.

Undoubtedly, the biggest challenge in the light curve fitting in this work was the treatment of spots and their influence on the resulting binary parameters. Several systems in our sample show stellar spots that are not only prominent, but also change rapidly, with the time scale of single orbital periods. This evolution is clearly seen in high-precision space-borne data (see also: Gillen et al. 2017). This means that the observed pattern of spots varies between two consecutive orbits, and is not stable during one orbital cycle (Figure 1, left). In other words, the binary appears different at the beginning and end of a single period. The scheme of light curve fitting typically requires (and assumes) that the studied system is stable during at least one orbital cycle, but one can see that it is not always the case.

One should also keep in mind, that the model of spots used in various light curve fitting codes (if incorporated) is simplified. A single cold spot is usually represented by a circular area of lower surface brightness, parametrised by its size, position, and contrast with respect to the "clear" photosphere. Multiple spots are often used in the analysis. The problem of modelling a spot distribution on a two-dimensional surface into a one-dimensional light curve is, however, ill-posed, i.e. there is more than one model that reproduces the observed light curve (e.g. Windmiller et al. 2010; Ioannidis \& Schmitt 2016; Basri \& Shah 2020), and different spot distributions (e.g. changing spot's location from primary to secondary) leads to variations in resulting stellar parameters at the level of several per-cent (e.g. Windmiller et al. 2010; Hełminiak et al. 2011). Even under this simplified spot model, the basic parameters are degenerated, i.e. change in spot's size can have the same effects on the light curve as the change in its temperature and/or latitude (Lanza et al. 2004; Ioannidis \& Schmitt 2016). In some cases, the use of multi-band photometry could help, but it would have to be taken simultaneously, and the current space photometric missions do not have such capabilities. Additionally, the observational data suggest that a significant population of short-period DEBs, which should be tidally locked, shows differences in rotation velocity with latitude (Lurie et al. 2017).

To properly model the rapidly-varying spots, as seen in our cases, one needs a code that allows for time evolution of all the parameters, and most likely non-linear. The later versions of the Wilson-Devinney code (WD; Wilson \& Devinney 1971; Wilson 2012) allow for migration of spots in longitude and their growth and decay (spot aging) in size. Still, the modelling process remains complicated, requires a lot of attention and time (as different distributions of spots need to be tested), does not allow for latitudal differential rotation, and possibility of failure (no convergence if starting from inaccurate values, or finding the local minimum) is high.

On top of that, the shape of the out-of-eclipse modulation can also be altered by the detrending scheme that was used in the data preparation (Figure 1, right). For example, a gradual drop in brightness, that was in fact introduced by the algorithm, could be mistaken with the increase of the spot's size, or decrease of its surface brightness. On the other hand, artificial "flattening" of the light curve leads to false stability of the parameters, and causes the loss of information about the true intrinsic variation. Inaccurate assumptions about distribution of spots and, simultaneously, instrumental effects may lead to, for example, incorrect depths of eclipses, which would affect temperature ratios, fractional radii and inclination.

In case of space-borne photometry of heavily spotted DEBs, one deals with a combination of a priori unknown effects, both intrinsic and artificial, and can not be sure how the resulting parameters of spots (and stars) were affected. Even if the migration and aging options are included (like in the WD), one still has to deal with several issues: (i) the size-temperature(-latitude) correlation; (ii) unknown number and location of spots; (iii) possible differential rotation; (iv) imperfect detrending; (v) time required. In such case it is very difficult to assess reliable uncertainties of the results. To our knowledge, there are no light curve fitting codes that allow to undertake this kind of analysis in a satisfactory and efficient way. A promising way to overcome this may be modelling the activity signals with Gaussian Processes (GP), implemented for example in Gillen et al. (2014), Gillen et al. (2017) or Smith et al. (2021). The advantage is that almost any out-of-eclipse modulation can be modelled simultaneously with the eclipsing binary, and uncertainties of the GP regression can be propagated to the stellar parameters. The dynamical character of the spots is therefore accounted for without modelling a spot per se, and at least partially, included in the error budget. It is, however, not clear, if the ambiguity of the process (i.e. applying the GP to the total brightness of the system, instead of just one component that could have spots) is also properly reflected in the final errors of stellar parameters.

For these reasons we decided to apply a different approach, with similar foundations as the one by Gillen et al. (2017). We do not try to model the spots as physical objects, but instead we embrace the fact that we have our results affected by systematics, and focus on the proper and reliable determination thereof in a time-efficient way. We base our approach on the fact that the spots evolve quickly, and the light curve looks different after single orbital periods.

As mentioned before, $K 2$ data were often divided into shorter parts, to easier deal with the evolution of spots, changes in the level of $l_{3}$, or other instrumental effects. Dividing data into subsets has been used or suggested as a robust way to obtain parameter errors by e.g. Maxted (2018); Maxted et al. (2020) or Hełminiak et al. (2019b). The Jктевор v40 is capable of fitting a number of polynomials (up to the 5th order) and sine functions to compensate for variations in some of the parameters (e.g. $\left.l_{1}, l_{2}, l_{\text {tot }}\right)$. We used these 

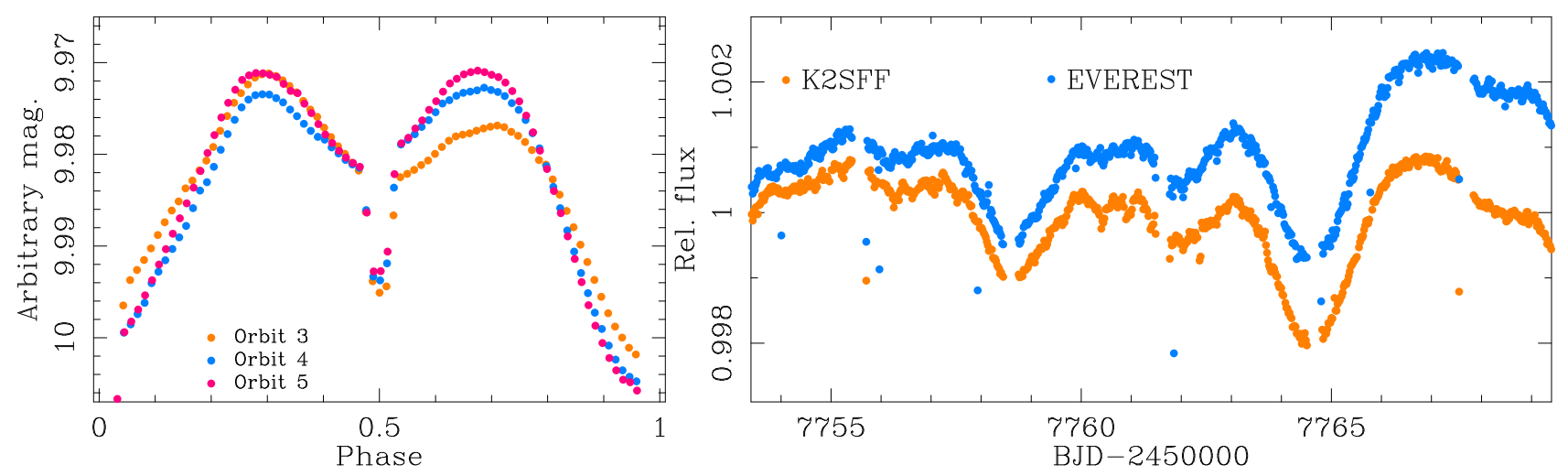

Figure 1. Left: A fragment of the C13 light curve of A-045, limited to three orbital periods (4.96 d), phase-folded, and zoomed to better show the evolution of the out-of-eclipse modulation. Each orbit is shown with a different colour. The change in the spot-originated modulation between consecutive orbits is clearly seen. Right: Comparison of two HLSP products (before the removal of outliers) for C12 LC data of A-231, also zoomed to better show the out-of-eclipse modulation. The use of different algorithms resulted in a different shape of the light curve, which may lead to different results when fitting for spots (e.g. the drop in $\mathrm{K} 2 \mathrm{sFF}$ flux could be interpreted as a drop in spot's temperature, increase of its size, or its migration closer to the equator).

features to model the dynamical out-of-eclipse variations coming from spots and long-term instrumental trends, as well as putative pulsations. The sines were found through an iterative process. After each JктевоР run, a Lomb-Scargle periodogram of the residuals was made, and the most prominent frequency was identified. The next fit included a sine function with the recently found frequency. If the new fit was better than the previous (in terms of $\mathrm{rms}$ ), the frequency was kept in the model, otherwise the second most prominent frequency was taken. These steps were repeated until no further advance in the quality of the overall model was noted. The number of sines and polynomials used varied between targets and parts. The exact values of the polynomial coefficients, and sine periods and amplitudes are given in Table B2 of the Appendix.

Parameter errors were estimated in two steps. First, for each single part we either used the the Monte-Carlo (MC) method or the residual-shift (RS) approach (Southworth et al. 2011), both available in JКтевор (tasks 8 and 9, respectively). The MC was preferred for light curves highly variable in time (like A-045 or A-085), while the RS was applied for more stable cases (A-060 or A-171) where short-term instrumental effects contribute more. These errors were adopted as final ones when single cadence/part was analysed (i.e. A-060, A-163, and A-171), or used for weighting, in case of multiple cadences/parts. In such cases, the adopted error was created by adding in quadrature the formal error of the weighted average and the rms of individual values (Hełminiak et al. 2015). In general, when the light curve shape changes rapidly the rms term dominates over the average, and vice versa for stable cases. In this way we take into account the influence of changes in the shape of the light curve (of any origin) into the uncertainties of a given parameter.

The only exception to the scheme above were the errors of $P$ and $T_{0}$, which were found with the MC option (task 8) used on complete sets of data.

\subsection{Spectra disentangling}

We used our spectroscopic data and run a disentangling procedure in order to obtain separate spectra of the components, suitable for further direct determination of effective temperatures and metallicities. We applied the version 3 of the code FDBINARY (FD3; Ilijić et al.
$2004)^{9}$, which performs separation of spectra in the Fourier space. This code has been chosen because it is capable of disentangling three components, which was needed in the case of A-045. Because FD3 uses component fractional intensities as input, we performed the separation in a relatively small wavelength range, where the flux ratio is roughly constant. We chose the range 5000-5500 $\AA$, rich in stellar spectral features, not affected by tellurics, and in which the SNR of individual spectra was relatively good. At the end, the SNR for each component spectrum was evaluated on the basis of the SNRs of individual observations, and intensity ratio of components (from TODCOR).

In the FD3 runs we did not combine spectra from different instruments. In particular, HIDES data were used for A-045 and A-083 = RU Cnc, CHIRON-fiber for A-060 and A-085, and FEROS for A-163, A171, and A-231. The remaining A-111 = FM Leo was not treated with this scheme, as its disentangled spectra (from CHIRON-slicer) were obtained by Sybilski et al. (2018) with the tomographic approach described in Konacki et al. (2010).

\subsection{Spectroscopic analysis}

To obtain individual effective temperatures, and systemic metallicities from the decomposed spectra, we used the v2020.10.01 version of the freely distributed code ISPEC (Blanco-Cuaresma et al. 2014). Flux errors were introduced on the basis of the previously calculated SNR. In this way we made sure the resulting uncertainties are trustworthy, which was verified by the reduced $\chi^{2}$, given in the output.

To find the atmospheric parameters we used the spectral synthesis approach, utilising the code SPECTRUM (Gray \& Corbally 1994), the MARCS grid of model atmospheres (Gustafsson et al. 2008), and solar abundances from Grevesse et al. (2007). ISPEC synthesizes spectra only in certain, user-defined ranges, called "segments". We followed the default approach, where these segments are defined as regions $\pm 2.5 \AA$ around a certain line. We decided to synthesize spectra around a set of lines carefully selected in such way, that various spectral fitting codes reproduce consistent parameters from a reference solar spectrum (Blanco-Cuaresma et al. 2016).

We run fits with the following parameters set free: effective

${ }^{9}$ http://sail.zpf.fer.hr/fdbinary/ 
temperature $T_{\text {eff }}$, metallicity [M/H], alpha enhancement $[\alpha / \mathrm{Fe}]$, and microturbulence velocity $v_{\text {mic }}$. The resolution $R$ was always fixed to a value appropriate for a given instrument, and gravity $\log (g)$ to the value corresponding to absolute values of mass and radius (see next Section), which is more precise than $\log (g)$ found from spectroscopy. The rotational velocity $v \sin (i)$ was also fixed and set to values expected from the synchronous rotation, which is expected for short-period circular or nearly circular orbits, as typically in our sample. In two cases, however - A-060 and A-163 - we set $v \sin (i)$ free, for reasons that will be explained later in the text. The macroturbulence velocity $v_{\text {mac }}$, which degenerates with rotation, was at all times calculated on-the-fly by ISPEC from an empirical relation.

As final values of systemic $[\mathrm{M} / \mathrm{H}]$ and $[\alpha / \mathrm{Fe}]$ we adopted averages of values obtained from each component. As their conservative uncertainties we added in quadrature the average formal parameter errors from ISPEC and standard deviation of the individual results. It is worth noting, that the values of $[\alpha / \mathrm{Fe}]$ were all formally indifferent from zero. In one case - A-171, the hottest system in our sample - the $[\alpha / \mathrm{Fe}]$ was found to be $-1.21 \pm 1.03 \mathrm{dex}$, which we find suspiciously low. Therefore we repeated the analysis with $[\alpha / \mathrm{Fe}]$ fixed at 0 .

In case of A-043 and A-085 we could also analyse spectra other than of the two eclipsing components. For the former, we actually run ISPEC on the primary and tertiary components only, as the secondary contributed less than $1 \%$ to the total flux, and the SNR of the disentangled spectrum was only $\sim 2$. For the latter, we also obtained one CHIRON-fiber observation during the total primary eclipse (when only the secondary's light was recorded). Additionally, early CORALIE and HDS observations of the visual companion were also used. In ISPEC runs for both tertiaries the $\log (g)$ parameter was set free. For both A-045 and A-085, the final values of systemic $[\mathrm{M} / \mathrm{H}]$ and $[\alpha / \mathrm{Fe}]$ were derived from all available spectra.

\subsection{Absolute stellar parameters}

The absolute values of stellar parameters were calculated with the JKTABSDIM ${ }^{10}$ procedure, which is available with JкTEвор. This code combines the output of spectroscopic and light curve solutions to derive a set of stellar absolute dimensions, related quantities, and distance, if effective temperatures are given. For this purpose the code uses the apparent, total magnitudes of a given binary in any of the $U, B, V, R, I, J, H, K$ bands. It compares the observed (total) magnitudes with absolute ones, calculated using a number of bolometric corrections (Bessell et al. 1998; Code et al. 1976; Flower 1996; Girardi et al. 2002), and surface brightness- $T_{\text {eff }}$ relations from Kervella et al. (2004). Flux ratios may also be used to further constrain individual absolute magnitudes of each component.

Apart from stellar, photometric, and orbital parameters, JKTABSDIM also calculates the rotation velocities predicted for the case of synchronisation of rotation with orbital period $v_{\text {syn }}$, the time scale of such synchronisation and the time scale of circularisation of the orbit.

\subsection{Isochrones}

The age of each system, and evolutionary status of each star were estimated with a grid of isochrones generated using a dedicated

10 http://www.astro.keele.ac.uk/jkt/codes/jktabsdim.html web interface, ${ }^{11}$ based on the Modules for Experiments in Stellar Astrophysics (MESA; Paxton et al. 2011, 2013, 2015, 2018), and developed as part of the MESA Isochrones and Stellar Tracks project (MIST v1.2; Choi et al. 2016; Dotter 2016). For the majority of the star's evolution in a DEB, the only interaction between the components is through gravity, which becomes important for small separations, leading to tidal interactions and consecutive phenomena (synchronisation, alignment, faster rotation, etc). The MIST models include rotation by default, but do not incorporate the MESAbinARY module, that is capable of evolving structure of two stars (Paxton et al. 2015). However, for the main sequence, early RGB phases and masses well below $2 \mathrm{M}_{\odot}$, the differences in stellar parameters are smaller than our measurement errors, and do not affect the resulting ages significantly. The activity may be, however, more relevant, at least for some of our objects (like A-045), as it can strongly affect the radii and effective temperatures of lowmass and sub-giant stars. The most recent isochrones for active stars (Somers et al. 2020) can more reliably reproduce those properties. We therefore conclude that in at least one case - A-045 - our age determination might be affected by the activity level.

The grid of isochrones was generated for iron abundance $[\mathrm{Fe} / \mathrm{H}]^{12}$ values from -4.0 to 0.50 dex with 0.05 dex steps, as well as for ages $10^{8.6}$ to $10^{10.2} \mathrm{Gyr}$, in logarithmic scale, every $\log (\tau)=0.01$.

On each isochrone we were looking for a pair of points that simultaneously best reproduce the observed masses $M_{1,2}$, radii $R_{1,2}$, and effective temperatures $T_{\text {eff } 1,2}$ of two components, as well as their flux ratio $l_{2} / l_{1}$ in the Kepler band, metallicity [Fe/H], and GEDR3 distance. The reddening-free distance $d_{0}$ was estimated simultaneously with the reddening $E(B-V)$, using the available observed total magnitudes in different filters, and the predicted total brightness of the system in the same filters, for a given pair of points (= stellar masses). Distances in each available band $d_{\lambda}$ were calculated from $T_{\mathrm{eff}}$-surface brightness relations from Kervella et al. (2004). Then, they were transformed to distance moduli $(m-M) \lambda$ with the standard relation $(m-M)=5 \log (d)+5$. Due to the interstellar extinction and reddening, individual values of $(m-M) \lambda$ were obviously not in agreement. To obtain the extinction-free modulus $(m-M)_{0}$ we fitted a straight line on the $A_{\lambda}$ vs. $(m-M) \lambda$ plane, where the $A_{\lambda}$ are extinction coefficients in each band. We followed the extinction law of Cardelli et al. (1989) $A_{U}: A_{B}: A_{V}: A_{R}: A_{I}: A_{J}: A_{H}:$ $A_{K}=4.855: 4.064: 3.1: 2.545: 1.801: 0.88: 0.558: 0.36$, which assumes $R_{V}=3.1$. The slope of the fitted line in this approach is the reddening $E(B-V)$, while the intercept is the extinction-free modulus $(m-M)_{0}$, which can be translated into the distance $d_{0}$. Additionally, when spectroscopic results for the tertiaries were available (i.e. their $T_{\text {eff }}$ and $\log (g)$ ), we also verified if they are well reproduced by the isochrone. This allowed us to constrain their other properties, like masses, radii, and evolutionary status.

It is worth noting that, like any other measurement, the $[\mathrm{M} / \mathrm{H}]$ values from ISPEC are uncertain, thus looking for the best-fitting isochrone, while keeping $[\mathrm{M} / \mathrm{H}]$ fixed, is not the most optimal approach. The values of $[\mathrm{Fe} / \mathrm{H}]$ (assumed equal to $[\mathrm{M} / \mathrm{H}]$, since $[\alpha / \mathrm{Fe}]=0)$ given as the result of isochrone fitting, may not be the same as those found in ISPEC. Hereafter, $[\mathrm{M} / \mathrm{H}]$ refers to the ISPEC results, while $[\mathrm{Fe} / \mathrm{H}]$ to isochrones.

11 http://waps.cfa.harvard.edu/MIST/

12 It is reasonable to assume that without significant deviations from solar amounts of $\alpha$-elements, the iron abundance $[\mathrm{Fe} / \mathrm{H}]$ sufficiently approximates the amount of elements metals $[\mathrm{M} / \mathrm{H}]$. 


\section{RESULTS}

Our final models are presented in Figures 2 (RVs) and 3 (light curves). The best-fitting isochrones are shown together with our measurements on the $M-R$ and $M-T_{\text {eff }}$ planes in Figure 4. The results, including orbital, physical, and atmospheric stellar parameters, as well as age and $E(B-V)$, are listed in Table 3 .

Below we briefly discuss our results for each system separately.

\section{$5.1 \quad \mathbf{A - 0 4 5}$}

This old, ( 9.3 Gyr) but highly active binary is composed of a nearly-solar-mass primary and a low-mass secondary. This is in general an interesting configuration - such pairs, composed of two vastly different stars, allow for more stringent tests of stellar evolution models than pairs of nearly identical components. For a given metallicity, their ages are tightly constrained by their masses and radii. 
Table 3. Orbital, physical, and atmospheric parameters of the studied systems. Values that were fixed and automatically calculated with empirical calibrations, are denoted by "fix" and "emp", respectively.

\begin{tabular}{|c|c|c|c|c|c|c|c|c|}
\hline $\begin{array}{l}\text { ASAS ID } \\
\text { EPIC } \\
\text { Running name }\end{array}$ & $\begin{array}{c}045021+2300.4 \\
247605441 \\
\text { A-045 }\end{array}$ & $\begin{array}{c}060505+2032.1 \\
202073040 \\
\text { A-060 }\end{array}$ & $\begin{array}{c}083730+2333.7 \\
212173112 \\
\text { A-083 (RU Cnc) }\end{array}$ & $\begin{array}{c}085002+1752.5 \\
211839462 \\
\text { A-085 }\end{array}$ & $\begin{array}{c}111245+0020.9 \\
201488365 \\
\text { A-111 (FM Leo) }\end{array}$ & $\begin{array}{c}163903-2847.2 \\
202674012 \\
\text { A-163 }\end{array}$ & $\begin{array}{c}171750-1915.3 \\
234440875 \\
\text { A-171 }\end{array}$ & $\begin{array}{c}231922-0852.2 \\
246024234 \\
\text { A- } 231\end{array}$ \\
\hline$P(\mathrm{~d})$ & $1.653362(7)$ & $2.1212341(35)$ & $10.1729311(55)$ & $5.22569411(9)$ & $6.728606(2)$ & $23.309595(35)$ & $3.136930(13)$ & $6.062036(4)$ \\
\hline$T_{0}(\mathrm{JD}-2450000)^{a}$ & $7020.57260(11)$ & $6768.11068(4)$ & $7747.34574(14)$ & $7143.61747(14)$ & $6812.22376(1)$ & $6909.36019(12)$ & $7660.70357(3)$ & $7743.442277(32)$ \\
\hline$T_{P}(\mathrm{JD}-2450000)^{b}$ & $7021.8138(21)$ & 6678.4861(14) & $7053.0440(35)$ & $6677.2237(06)$ & $7429.5730(3)$ & 6081.11(10) & $6427.1050(4)$ & 6106.92(37) \\
\hline$K_{1}\left(\mathrm{~km} \mathrm{~s}^{-1}\right)$ & $60.33(25)$ & 123.7(1.0) & $70.62(9)$ & $94.86(5)$ & $76.033(19)$ & 45.44(3) & $100.66(12)$ & $70.59(5)$ \\
\hline$K_{2}\left(\mathrm{~km} \mathrm{~s}^{-1}\right)$ & $137.7(1.0)$ & $125.1(6)$ & $67.2(3)$ & 79.99(8) & $78.646(22)$ & $56.56(7)$ & $101.55(8)$ & 79.71(11) \\
\hline$\gamma_{1}\left(\mathrm{~km} \mathrm{~s}^{-1}\right)$ & $-29.0(2)$ & $20.7(5)$ & $2.55(9)$ & $2.73(4)$ & $12.527(51)$ & $9.70(5)$ & $-40.76(6)$ & $4.90(6)$ \\
\hline$\gamma_{2}-\gamma_{1}\left(\mathrm{~km} \mathrm{~s}^{-1}\right)$ & $6.8(1.0)$ & 0.0 (fix) & $0.2(3)$ & $0.0($ fix $)$ & $0.036(33)$ & $0.0($ fix $)$ & $0.0($ fix $)$ & $0.21(22)$ \\
\hline$q$ & $0.4383(37)$ & $0.989(9)$ & $1.050(4)$ & $1.1859(14)$ & $0.9668(4)$ & $0.8033(12)$ & $0.9912(13)$ & $0.8855(14)$ \\
\hline$M_{1} \sin ^{3}(i)\left(\mathrm{M}_{\odot}\right)$ & $0.924(17)$ & $1.702(22)$ & $1.347(11)$ & $1.324(3)$ & $1.3119(8)$ & $1.420(4)$ & $1.3495(26)$ & $1.131(3)$ \\
\hline$M_{2} \sin ^{3}(i)\left(\mathrm{M}_{\odot}\right)$ & $0.405(5)$ & $1.683(27)$ & $1.415(7)$ & $1.570(2)$ & $1.2683(7)$ & $1.140(2)$ & $1.3376(32)$ & $1.002(2)$ \\
\hline$a \sin (i)\left(\mathrm{R}_{\odot}\right)$ & $6.472(34)$ & $10.43(5)$ & $27.73(6)$ & $18.066(10)$ & $20.573(4)$ & 46.99(4) & $12.541(8)$ & $18.015(14)$ \\
\hline$e$ & 0.0 (fix) & 0.0 (fix) & 0.0 (fix) & 0.0 (fix) & 0.0 (fix) & $0.026(2)$ & $0.0030(15)$ & $0.0049(33)$ \\
\hline$\omega\left(^{\circ}\right)$ & - & - & - & - & - & 259(1) & $272(2)$ & 103(12) \\
\hline$r_{1}$ & $0.1629(34)$ & $0.1957(4)$ & $0.0789(15)$ & $0.0869(13)$ & $0.07900(43)$ & $0.04588(12)$ & $0.1334(5)$ & $0.0909(5)$ \\
\hline$r_{2}$ & $0.0628(14)$ & $0.1919(5)$ & $0.1781(26)$ & $0.1783(24)$ & $0.07293(58)$ & $0.02603(8)$ & $0.1312(5)$ & $0.0615(13)$ \\
\hline$i\left(^{\circ}\right)$ & $85.26(36)$ & $85.81(2)$ & $89(1)$ & $88.1(7)$ & $87.939(28)$ & $88.668(14)$ & $86.486(14)$ & 87.01(19) \\
\hline$J$ & $0.08(2)$ & $0.996(4)$ & $0.205(12)$ & $0.624(21)$ & $0.996(7)$ & $0.965(12)$ & $0.981(8)$ & $0.892(33)$ \\
\hline$l_{2} / l_{1}$ & $0.011(3)$ & $0.954(9)$ & $1.10(8)$ & $2.58(15)$ & $0.843(22)$ & $0.3112(27)$ & $0.975(11)$ & $0.405(17)$ \\
\hline$l_{3} / l_{\mathrm{tot}}{ }^{c}$ & 0.0 (fix) & $0.016(3)$ & 0.0 (fix) & variable & $0.007(4)$ & $0.013(7)$ & $0.051(4)$ & $0.031(19)$ \\
\hline$r m s_{\mathrm{RV} 1}\left(\mathrm{~km} \mathrm{~s}^{-1}\right)$ & 0.83 & 0.97 & 0.23 & 0.09 & 0.051 & 0.10 & 0.54 & 0.082 \\
\hline$r m s_{\mathrm{RV} 2}\left(\mathrm{~km} \mathrm{~s}^{-1}\right)$ & 3.71 & 0.65 & 0.90 & 0.13 & 0.084 & 0.22 & 0.26 & 0.147 \\
\hline$r m s_{\mathrm{LC}}(\mathrm{mmag})$ & 0.97 & 0.84 & 1.1 & 0.28 & 0.16 & 0.19 & 0.33 & 0.20 \\
\hline$M_{1}\left(\mathrm{M}_{\odot}\right)$ & $0.934(17)$ & $1.716(22)$ & $1.347(11)$ & $1.326(3)$ & $1.3144(8)$ & $1.421(4)$ & $1.3571(26)$ & $1.136(3)$ \\
\hline$M_{2}\left(\mathrm{M}_{\odot}\right)$ & $0.409(5)$ & $1.697(28)$ & $1.415(7)$ & $1.573(3)$ & $1.2707(7)$ & $1.141(2)$ & $1.3452(32)$ & $1.006(2)$ \\
\hline$a\left(\mathrm{R}_{\odot}\right)$ & $6.494(35)$ & $10.46(5)$ & $27.73(6)$ & $18.076(12)$ & 20.591(4) & $47.00(4)$ & $12.564(9)$ & $18.040(15)$ \\
\hline$R_{1}\left(\mathrm{R}_{\odot}\right)$ & $1.058(23)$ & $2.048(10)$ & $2.188(42)$ & $1.571(25)$ & $1.627(9)$ & $2.157(6)$ & $1.676(7)$ & $1.640(9)$ \\
\hline$R_{2}\left(\mathrm{R}_{\odot}\right)$ & $0.408(9)$ & $2.008(11)$ & $4.939(73)$ & $3.222(42)$ & $1.498(12)$ & $1.224(4)$ & $1.648(6)$ & $1.110(23)$ \\
\hline $\log \left(g_{1}\right)$ & $4.360(18)$ & $4.050(3)$ & $3.888(17)$ & $4.168(14)$ & $4.134(5)$ & $3.923(2)$ & $4.122(3)$ & $4.064(5)$ \\
\hline $\log \left(g_{2}\right)$ & $4.829(19)$ & $4.062(4)$ & $3.202(13)$ & $3.619(11)$ & $4.191(7)$ & $4.320(3)$ & $4.133(3)$ & $4.350(18)$ \\
\hline$v_{\mathrm{rot}, 1}\left(\mathrm{~km} \mathrm{~s}^{-1}\right)^{d}$ & $32.4(7) \mathrm{s}$ & $39.7(6.6) \mathrm{i}$ & $10.9(2) \mathrm{s}$ & $15.21(24) \mathrm{s}$ & $12.23(7) \mathrm{s}$ & $14.1(7) \mathrm{i}$ & $27.03(10) \mathrm{s}$ & $13.69(7) \mathrm{s}$ \\
\hline$v_{\mathrm{rot}, 2}\left(\mathrm{~km} \mathrm{~s}^{-1}\right)^{d}$ & $12.5(3) \mathrm{s}$ & $35.3(6.2) \mathrm{i}$ & $24.6(4) \mathrm{s}$ & $31.18(42) \mathrm{s}$ & $11.26(9) \mathrm{s}$ & $5.7(5.8) \mathrm{i}$ & $26.57(10) \mathrm{s}$ & $9.26(19) \mathrm{s}$ \\
\hline$T_{\mathrm{eff}, 1}(\mathrm{~K})$ & $5668(71)$ & $7405(432)$ & $6569(84)$ & 6554(207) & 6371(115) & $6630(83)$ & $6530(130)$ & $6263(41)$ \\
\hline$T_{\mathrm{eff}, 2}(\mathrm{~K})$ & $3590(100)^{e}$ & $7407(382)$ & 4761(147) & $5730(250)$ & $6353(116)$ & $6586(380)$ & $6501(142)$ & $6240(99)$ \\
\hline$[M / H]^{f}$ & $-0.26(26)$ & $-0.12(21)$ & $-0.26(34)$ & $-0.01(27)$ & $-0.13(9)$ & $-0.19(16)$ & $0.04(12)$ & $-0.39(7)$ \\
\hline$[\alpha / F e]$ & $0.03(63)$ & 0.00 (fix) & $-0.04(37)$ & $-0.02(10)$ & $0.10(23)$ & $0.03(15)$ & $-0.03(15)$ & $0.10(7)$ \\
\hline$v_{\mathrm{mic}, 1}\left(\mathrm{~km} \mathrm{~s}^{-1}\right)$ & $2.42(22)$ & $2.55(80)$ & $10.07(\mathrm{emp})$ & $1.45(31)$ & $1.60(19)$ & $2.16(14)$ & $2.27(31)$ & $1.91(9)$ \\
\hline$v_{\text {mic }, 2}\left(\mathrm{~km} \mathrm{~s}^{-1}\right)$ & - & $2.44(76)$ & $2.64(40)$ & $1.30(39)$ & $1.68(20)$ & $2.24(75)$ & $2.12(31)$ & $0.94(27)$ \\
\hline$E(B-V)^{g}(\mathrm{mag})$ & $0.132(21)$ & $0.163(62)$ & $0.201(24)$ & $0.004(3)$ & $0.054(22)$ & $0.139(13)$ & $0.217(24)$ & $0.037(11)$ \\
\hline$\tau(\mathrm{Gyr})^{e}$ & $9.3(1.5)$ & $1.00(15)$ & $3.02(40)$ & $2.40(28)$ & $2.72(31)$ & $2.40(30)$ & $2.29(34)$ & $4.68(57)$ \\
\hline
\end{tabular}

${ }^{a}$ Mid-time of the primary (deeper) eclipse. ${ }^{b}$ Time of pericentre or quadrature. ${ }^{c}$ Value obtained in the fit, not bearing a physical meaning (see Sect. 4.2) $d$ Marked with "s" for (pseudo-)synchronous rotation velocities, as given by JKTABSDIM, and with "i" for projected $v \sin (i)$ obtained with ISPEC.

${ }^{e}$ Estimated from isochrone fitting. ${ }^{f}$ From ISPEC. ${ }^{g}$ Value that reproduces the GEDR3 distance. 
The secondary contributes only about $0.5 \%$ of the total flux in the Kepler photometric band, therefore the observed very strong and rapidly evolving out-of-eclipse modulation (Fig. 3) is probably caused by spots located on the primary only. Its scale ( $\sim 66 \mathrm{mmag})$ is much larger than of the ellipsoidal variations ( $\sim 5 \mathrm{mmag})$, and the depth of the secondary eclipse $(\sim 12 \mathrm{mmag})$. The pattern of spots changes in time scales comparable to the short orbital period $(1.653 \mathrm{~d})$, meaning that every orbital revolution the light curves looks differently. A closer inspection shows that two brightness minima, presumably related to two different groups of spots, move in longitude with unequal rates, which would imply differential rotation of the primary. This obviously made the fit difficult, thus the resulting $r m s$ of the residuals is one of the highest in the sample, and the scatter significantly increases during eclipses. To model this system, we split the light curve to five pieces, used a single 5-th order polynomial, and up to seven sine functions per piece.

Moreover, the low brightness of the secondary makes it hard to detect in the spectra. Its RVs were not measured in some cases. An additional obstacle was a presence of strong third light in the spectra, which produced a narrow peak in the cross-correlation function (CCF), at a position near the centre-of-mass velocity $\gamma_{1}$ of the system. The rapidly rotating primary produces a broad CCF peak, which very often interfered with the narrow one from the third light, making its RV measurements difficult and uncertain. We estimate the RV of the third light to be $-28.1 \pm 0.4 \mathrm{~km} \mathrm{~s}^{-1}$, very close to $\gamma_{1}$. This suggests that the narrow CCF peak may come from a star gravitationally bound to the eclipsing pair.

However, we have found no significant third light $l_{3}$ in JKTEBOP fits. Thus, in the final runs we decided to hold $l_{3}$ fixed to 0 . The reason for this is most likely the additional flux correction adopted in the detrending scheme. Despite those difficulties we managed to obtain relatively good precision of $1.2-1.8 \%$ in masses, and $\sim 2.2 \%$ in radii.

In this special case the ISPEC analysis was done on the primary (SNR of the recovered spectrum $\sim 171$ ) and the tertiary (SNR 92). The latter was found to have $T_{\text {eff }}=4990(340) \mathrm{K}$ and $\log (g)=3.16(58)$ dex, which suggests it is a (sub-)giant, therefore it is more evolved and massive than the primary. The best-fitting isochrone was found for the age of $9.3 \mathrm{Gyr}$, and $[\mathrm{Fe} / \mathrm{H}]$ of $+0.05 \mathrm{dex}$, different from the ISPEC value only by $1.2 \sigma$. This isochrone predicts the tertiary to have $M \simeq 1.056 \mathrm{M}_{\odot}$ and $2.34<R<5.88 \mathrm{R}_{\odot}$.

A-045 is the only case in our sample, where the $T_{\text {eff }}$ of the secondary was not used in isochrone fitting. Instead, it was evaluated from the distribution of models falling within $3 \sigma$ from the other parameters. The value given in Table 3 is calculated from this distribution. It is drawn with grey symbols in the lower A-045 panel of Fig. 4.

We should, however, keep in mind that for reasons discussed in Sect. 4.6, our age estimation in this case may be affected by the primary's activity, i.e. the radius might have been inflated, $T_{\text {eff }}$ underestimated, and the whole system might be slightly younger than 9.3 Gyr.

\section{$5.2 \quad \mathrm{~A}-060$}

This binary is composed of two very similar, yet not identical stars. The mass ratio $q$ is differs from 1 by only $1.2 \sigma$, but the fractional radii $r_{1}$ and $r_{2}$ differ by nearly $7 \sigma$. This is due to relatively low precision of RV measurements, hampered mainly by fast rotation of both components. Despite that, the precision in masses is still good: 1.3 and $1.7 \%$ for the primary and secondary, respectively.

The entire light curve (Fig. 3) was modelled in one fit. Except ellipsoidal modulations ( $\sim 17 \mathrm{mmag}$ ) and a weak long-term trend, no significant out-of-eclipse variations have been detected, thus only a single 5-th degree polynomial (without any additional sine functions) had to be used. The spread of JKTEBOp fit residuals is rather large but roughly constant for every orbital phase. This allowed us to reach a very good precision in radii, at the level of $0.6 \%$ for both components.

The ISPEC analysis turned out pose some challenges, and resulted in large uncertainties. The two components were quickly found to be hotter than $7000 \mathrm{~K}$, and rotating rapidly. This, combined with the mediocre SNR of the disentangled spectra ( $\sim 66)$, made the spectral features shallow. In this case, taking into account the possible high values of temperatures, we decided to set the $v \sin (i)$ parameter free (the calculations of $v_{\text {syn }}$ implemented in JKTABSDIM are relevant for cooler stars with convective envelopes). Components of A-060 appear to rotate slower, than in case of synchronous rotation $\left(\sim 48.8 \mathrm{~km} \mathrm{~s}^{-1}\right)$.

Comparison with isochrones resulted in determination of the most probable age of $1.00 \mathrm{Gyr}$ for $[\mathrm{Fe} / \mathrm{H}]=0.0 \mathrm{dex}$. Both components are on the main sequence (MS), and evolved from the zero-age main sequence (ZAMS). The predicted temperatures, highest in our sample, are nearly identical, which can be deduced from the eclipses of almost the same depth.

An interesting feature of this system is that both components lay in the theoretical instability strip for $\delta$ Scuti type (dSct) pulsators. When compared to a sample of dSct pulsators in binaries, compiled by Kahraman Aliçavuş et al. (2017), one can see that parameters of both components of A-060 agree well with the sample. We can use the relations obtained by Kahraman Aliçavuş et al. (2017) between the period of pulsations $P_{\text {pul }}$ and various stellar and binary parameters $\left(P, M, R, T_{\text {eff }}, \log (g), q\right)$. The obtained values of $P_{\text {puls }}$ vary from $\sim 0.034 \mathrm{~d}$ (vs. $R$ ), up to $0.081 \mathrm{~d}$ (vs. $q$ ), with most of the values between 0.040 and $0.055 \mathrm{~d}$. With the current data we do not detect any significant pulsation signal, but this may be due to the cadence of observations, and/or features of the adopted detrending algorithm. A better insight may come from TESS observations, expected to be done in cycles 43 to 45 .

\subsection{A-083 = RU Cnc}

RU Cnc is a chromospherically active system of the RS CVn type. In its $K 2$ light curve (Fig. 3) we can see quickly evolving spots, flares, and a total primary (deeper) eclipse, with some variations in its depth. These characteristics are typical for a system with large, cold, heavily spotted component, accompanied by a smaller hot one. The scale of the spot-originated variability ( $\sim 80 \mathrm{mmag})$ overwhelms the ellipsoidal modulation ( $~ 8 \mathrm{mmag})$.

The CCF shows two clear peaks of significantly different widths. The RVs of the cold secondary are strongly affected by spots and rapid rotation, with the $r m s$ of $0.90 \mathrm{~km} \mathrm{~s}^{-1}$, while that of the hot primary have significantly better precision, with the $r m s$ of $230 \mathrm{~m} \mathrm{~s}^{-1}$. Both values are better than for the previous studies: Popper (1990) gives 4.3 and $4.4 \mathrm{~km} \mathrm{~s}^{-1}$ for the primary (hot) and secondary (cold), respectively, while Imbert (2002) analogously gives 1.80 and $3.22 \mathrm{~km} \mathrm{~s}^{-1}$. The final precision of mass determination in our solution is at a very good level of 0.8 and $0.5 \%$ for the primary and secondary, respectively. The ISPEC analysis of the deconvolved spectra (SNR $\sim 86$ and 58 for the primary and secondary, respectively) suggested a small depletion of metals, but with high uncertainties. As expected, the evolved inflated secondary turned out to be much cooler than the primary.

It is important to note that in his Table 2, Imbert (2002) proba- 

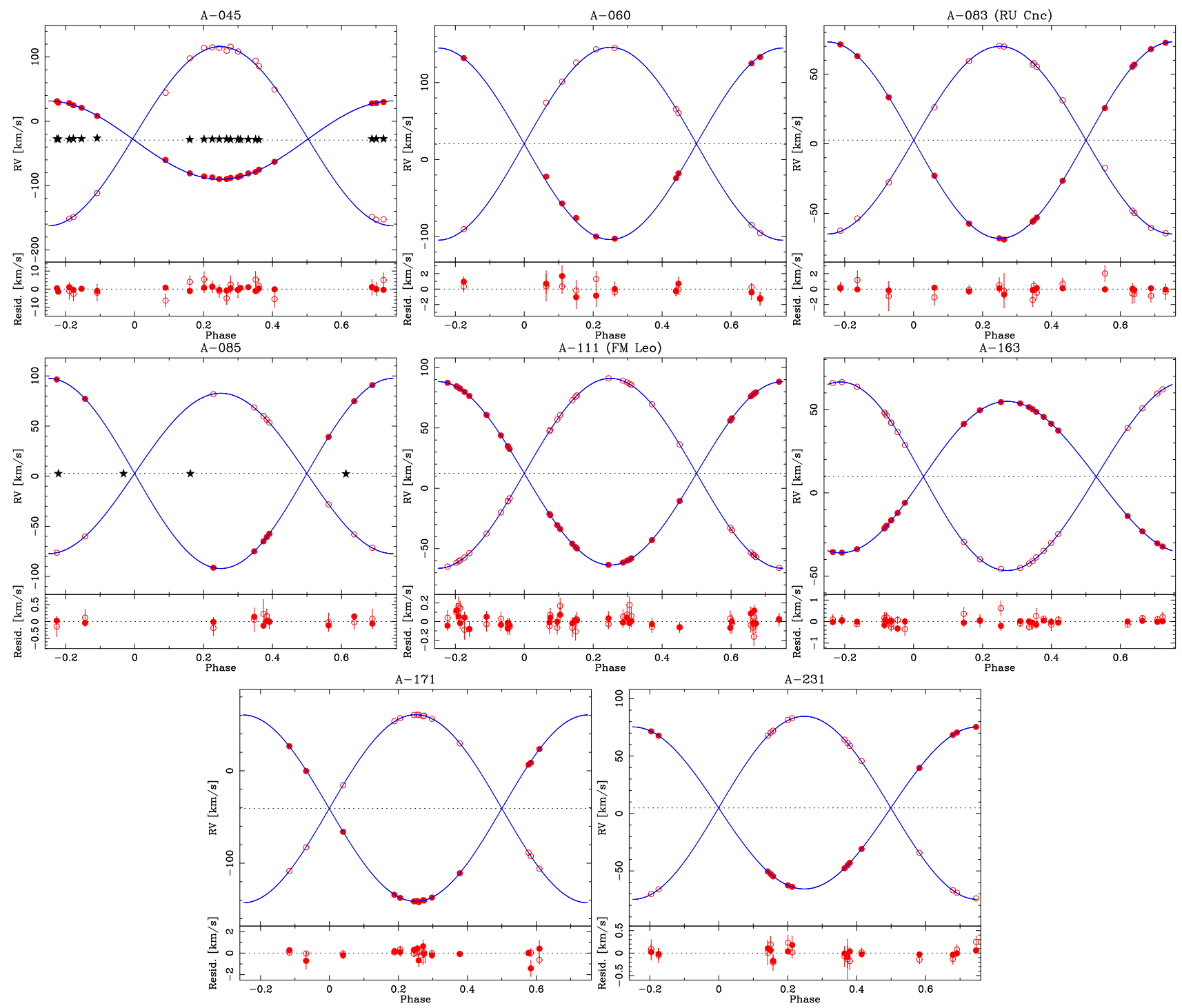

Figure 2. RV measurements (red points) and solutions (blue lines) for the studied systems, phase-folded with the orbital period, with phase 0 set to the moment of the primary eclipse. Filled points are for the primaries, and open for secondaries. Black dashed horizontal lines mark systemic velocities $\gamma$. Lower panels show residuals of fits. Black stars on the plots for A-045 and A-085 represent RVs of the companions to the DEB.

bly confused the hot and cold component, and this confusion might have been taken over by other authors. The hotter component (spectral type F5 therein) is shown to have larger mass than the cooler (K1IV). Notably, the assignment in Table 3 of the same work is different. The RV curves from Imbert's Figure 4, show that the hotter component is the one with more measurements (31) than the cooler K1IV-type component (21). It also seems to show larger RV amplitude (thus lower mass) and smaller spread ( $r m s)$. We confirmed this by taking the original measurements and running an orbital fit with v2FIT - the component with more data points is the one with larger $K$, and smaller $r m s$, which is in agreement with the scenario that it is less massive and slower rotating. Table 2 shows the situation opposite to Fig. 4, where more observations and smaller spread were attributed to the cold component, making it the more massive one. Additionally, a note in the SB9 catalogue (Pourbaix et al. 2004) points the hotter component (the primary) as the one with more measurements. Our new data also clearly show that the hot- ter, earlier type primary, which produces the narrow CCF peak and lower $r m s$ of the fit, is the less massive one (Fig. 2, Tab. 3).

We thus believe that Imbert unintentionally confused the components in Table 2 (but not Table 3 ) of his work, improperly making the hot one also the more massive one. It is correct at the Main Sequence, but that time it was already known that one of the stars is a giant. This probably led to further interpretations (like in Eggleton \& Yakut 2017) that the later-type giant star must have undergone a substantial mass loss. When the situation is inverted (the colder and larger companion is more massive, as in our solution), the inconsistency with evolutionary models vanishes (Fig. 4), and no additional assumption of mass loss or other processes need to be made.

The photometric LC data consist of two Campaigns, 5 and 18, which for the Јктевор analysis were split into 3 and 2 parts, respectively. We modelled the photometric trends and spot-originated variations with a single 5-th degree polynomial and between five and seven sine functions. The activity obviously affected the final 

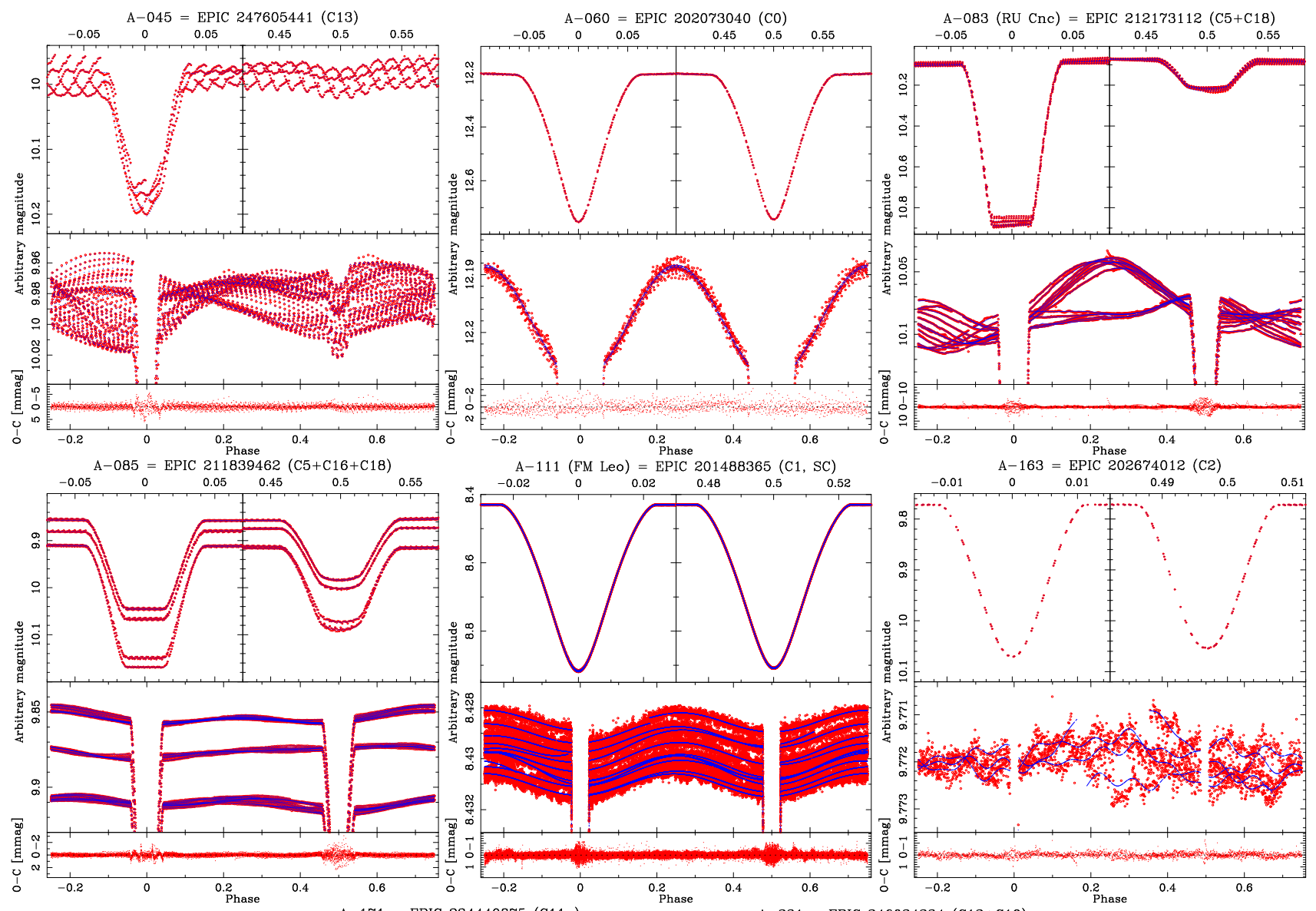

$\mathrm{A}-111(\mathrm{FM}$ Leo $)=$ EPIC $201488365(\mathrm{C} 1, \mathrm{SC})$
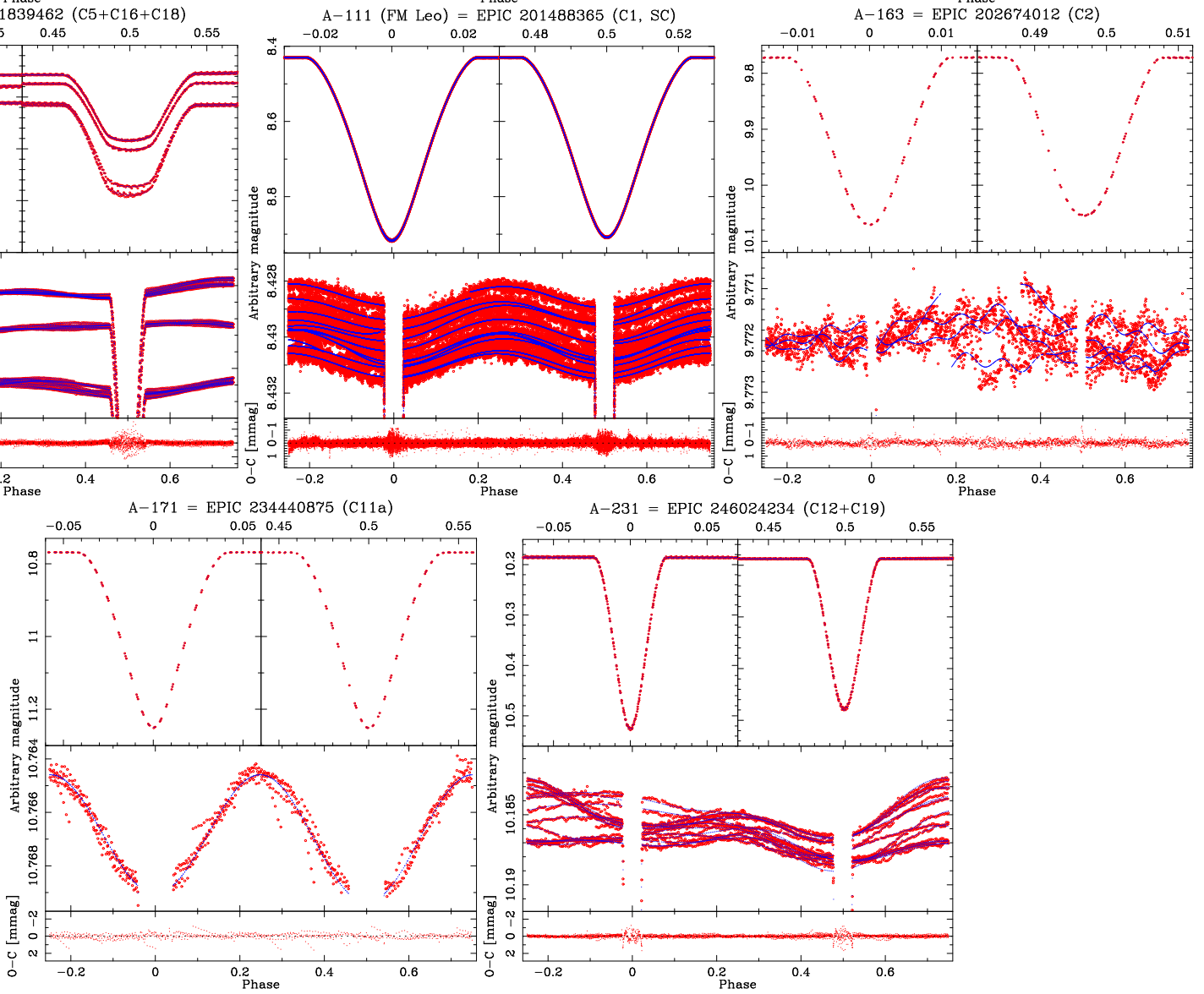

Figure 3. Observed (red) and modelled (blue) photometric $K 2$ data for the studied systems, phase-folded with the orbital period, with phase 0 set to the moment of the primary eclipse. For each system we show zooms on the primary and secondary eclipse (top), on the out-of-eclipse variations (middle), and residuals of the fit. Data for all available cadences are shown (cadence numbers given in labels). In A-045 the scale of spot-originated variation is larger than the secondary eclipse. Changes in pattern of spots in time is also clearly seen for A-231, A-083 and A-085. In the last case, changes in the total brightness and eclipse depths are caused by the amount of the companion's contribution varying from cadence to cadence, and within C16. The short-period fluctuations in A-163 may come from oscillations.

results, especially $r_{1,2}$ (thus $R_{1,2}$ ), whose uncertainties were dominated by the rms of partial results. Still, our approach resulted in relatively good final precision in absolute radii, at the level of 1.5-1.9\%.

A-083 is one of two systems in our sample, for which shortcadence (SC) data are available. Their closer examination reveals multiple discontinuities, very often during the primary eclipse (which makes it nearly impossible to properly assess its true depth), as well as high complexity of the spot pattern on the secondary. In Figure 5 we show a zoom on eight consecutive secondary eclipses recorded in C5 SC data (detrended with EVEREST, obtained directly from MAST, and cleaned of the outliers). They reveal multiple distortion events which we interpret as caused by the (smaller) primary transiting in front of inhomogeneities on the secondary's surface. Observations of such phenomena in eclipsing binaries are rare, but have been reported (e.g. KIC 10514158; Lurie et al. 2017). 

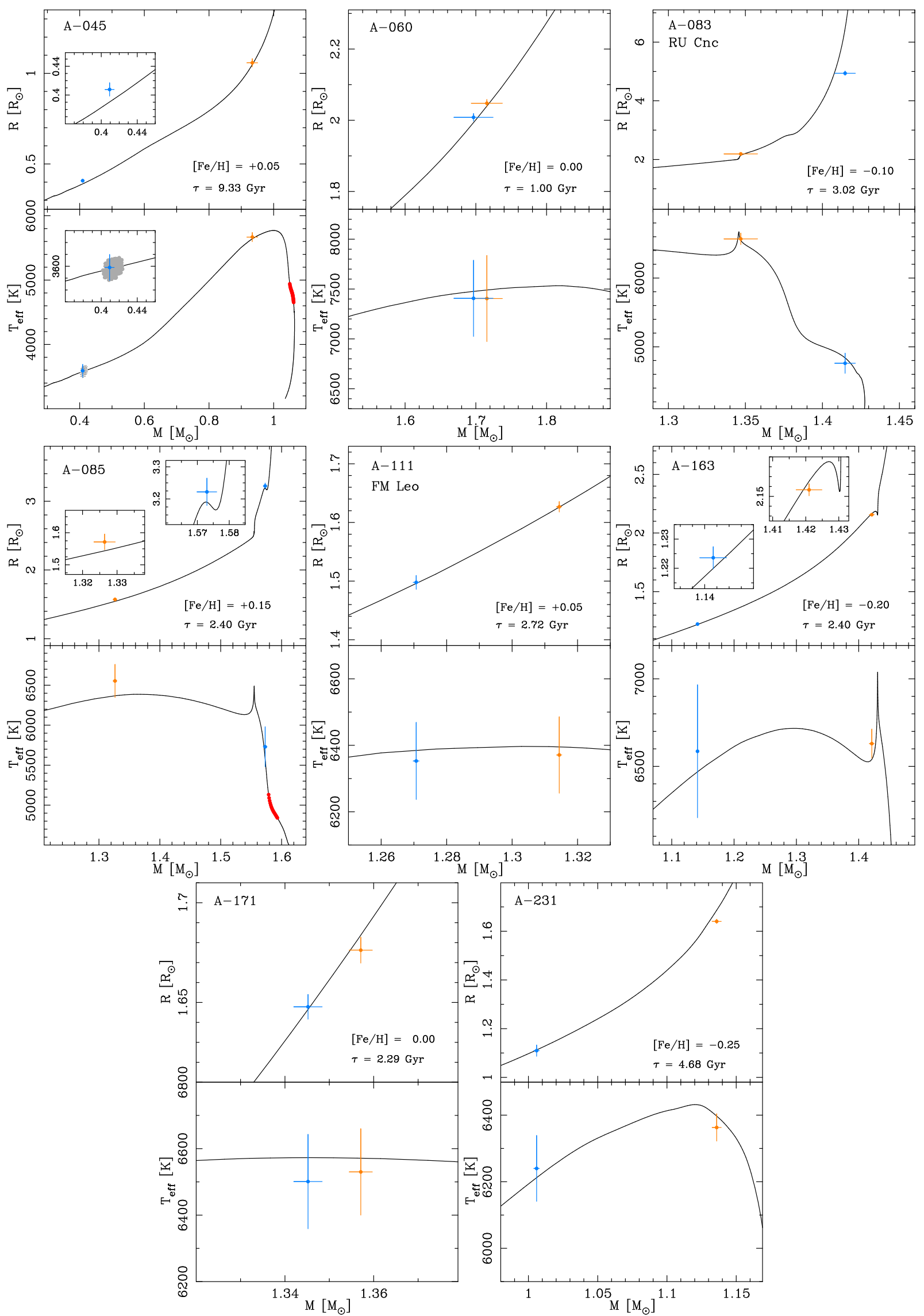

Figure 4. Comparison of masses, radii and effective temperatures with the best-fitting isochrones. Their ages and metalicities are labelled. Orange and blue points denote primary and secondary components, respectively. Red lines mark segments of given isochrones that reproduce the measured properties $\left(T_{\text {eff }}\right.$, log $g$ ) of tertiary companions to A-045 and A-085 (outside the panels in $M-R$ diagrams). 


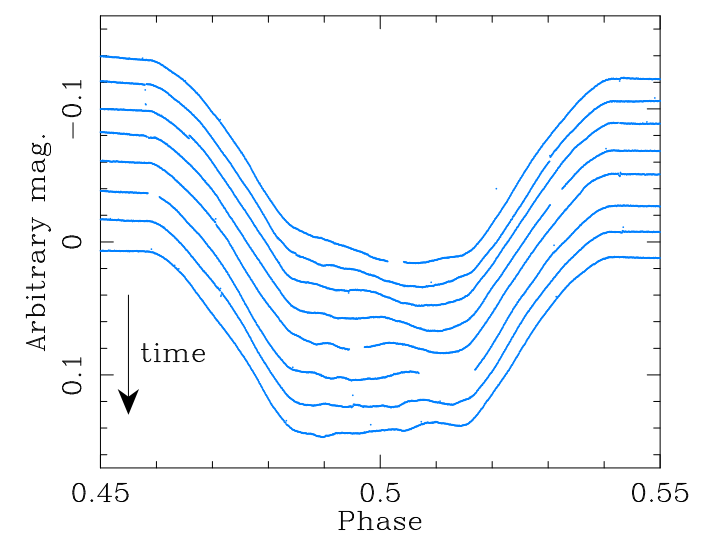

Figure 5. Variations in the shape of the secondary eclipse of A-083. Eight events from C5 (SC data)are shown folded in orbital phase, and arbitrarily shifted in such way, that the earliest is on the top. One can see distortions caused by the (smaller) primary component obscuring inhomogeneities on the secondary's surface. Variation in the eclipse's shape means that the location of those surface features changes from orbit to orbit.

Such rapid evolution of spots in time (also seen in A-045 or A085) makes analysis difficult and vulnerable to systematical errors. Çokluk et al. (2019) took the C5 long-cadence data, and found a solution with the PHOEBE code (Prša \& Zwitter 2005), where properties of spots (location, size, temperature contrast) can be modelled, but in their model the spots are stationary, making their solution unrealistic (as we argue in Section 4.2). Notably, Çokluk et al. did not make a full fit to the $\mathrm{C} 18$ data, but only changed the parameters of spots in order to reproduce the light curve. Additionally, their study lacks information about the details of the fitting scheme (e.g. treatment of effective temperatures and their influence on other parameters), quality of the fit, or even properly presented residuals thereof, which, from the inspection of their Figure 5, seem to be quite large and inhomogenous in phase, strongly suggesting systematical errors. Not much is said about the error budget, and, most likely, the systematics were not properly accounted for. All this makes the stellar parameters by Çokluk et al. (2019) unreliable, in terms of both accuracy and precision.

In comparison, our approach allows for dynamical changes of the combined detrending and spot-originated brightness modulation, however without modelling the spots themselves. This also introduces systematical uncertainties (for example, the model may predict incorrect eclipse depth which affects $r_{1,2}$ ), but we attempt to quantify them and incorporate into our uncertainties, as described in Sect. 4.2. More accurate results are still possible to obtain through a dedicated, detailed analysis, that focuses on the secondary eclipses and takes into account the non-static character of spots, i.e. is capable of modeling the evolution of spot parameters during a single orbital period, including possible differential rotation, and very careful detrending of the SC data, so the intrinsic variability of short time scales and small amplitudes is not lost. Such an effort was not in the scope of this work, and may be done in the future.

In Table 4 we compare our results with those of Popper (1990), Imbert (2002), and Çokluk et al. (2019). Masses and radii from the two former studies come from the assumption of $i=90^{\circ}$, and estimates of fractional radii from incomplete photometry. The most recent study uses RVs of both previous authors, and probably carries on the confusion on which component is hotter and more massive. One can see that our results are still the most precise ones, and probably also more accurate, while the treatment of errors is better.
We are surprised to see that, for example, RV amplitudes $K_{1,2}$ in Çokluk et al. (2019) have errors of only $80 \mathrm{~m} \mathrm{~s}^{-1}$, even though they were derived from data of substantially worse quality ( $r m s$ of single $\mathrm{km} \mathrm{s}^{-1}$ ) than ours. This again undermines the reliability of these results. Our study is also the first where temperatures were determined in a direct way.

Comparison of our measurements with the MESA isochrones points towards the age of $3.02 \mathrm{Gyr}$, and metallicity below solar. The primary is at the very end of its main sequence evolution, while the secondary is at the red giant branch, growing with an accelerating pace, currently about $0.02 \mathrm{R}_{\odot} \mathrm{Myr}^{-1}$. Using the formula from Eggleton (1983), we can estimate the effective radius of the secondary at the moment of reaching the Roche lobe to be $10.63 \mathrm{R}_{\odot}$. This should happen in about $110 \mathrm{Myr}$, assuming negligible loss of mass and angular momentum, however, as Çokluk et al. (2019) have convincingly shown, the orbital period of RU Cnc gradually decreases as $7.9(1.2) \times 10^{-7} \mathrm{~d} \mathrm{yr}^{-1}$. Considering this, and masses of both components, RU Cnc will likely evolve into a W UMa-type binary (Y1ldiz 2014).

The secondary resides in a very interesting place on the $M-R$ and $T_{\text {eff }}-\log (g)$ diagrams, ${ }^{13}$ in the so-called Hertzsprung gap, between old giants and sub-giants that have just moved from the main sequence, which is caused by a rapid growth in this phase. This gap is best seen on $\log (g)$ distribution, between values 3.5 and 3.0 dex. A star of $1.415 \mathrm{M}_{\odot}$ needs about $160 \mathrm{Myr}$ to go from $\log (g)=3.5$ to 3.0 dex, and the secondary of A-083 should reach that point in about $50 \mathrm{Myr}$. Till now, no component of a DEB with precisely measured properties has been found in this region, at least among the FGK spectral types.

RU Cnc gives a unique opportunity to study rare stages of stellar evolution, activity, and binary interactions but requires a special attention when comes to light curve modelling, that has not been applied to any other system so far. We have reached a good precision and accuracy in determination of stellar parameters, but there is still space for improvement, e.g. precise element abundances are missing. The Community is encouraged to study this unique system, especially its chemical composition, magnetic field, and future tidal evolution.

\section{$5.4 \quad$ A-085}

This system is similar to the previous one: the light curve shows a total primary minimum and out-of-eclipse variations that change in time (Fig. 3), the CCF is composed of a broad and a narrow peak, and the eclipsing pair is composed of a main sequence primary, and a sub-giant secondary. The main difference is that the depths of eclipses are more similar, indicating temperature ratio closer to 1 than for RU Cnc, and a strong and variable addition of the third light. This system has been observed in three campaigns - C5, C16, and $\mathrm{C} 18$ - during which the orientation of the satellite was varying. Each time the amount of flux from the visual companion, that was falling into the pixel aperture mask, was different. Especially, it changed drastically during C16, which is reflected by the change in depths of eclipses (the lowest of three A-085 curves in Fig. 3). For this reason, and also to model the varying out-of-eclipse modulation, likely coming from spots $(\sim 10 \mathrm{mmag})$ with addition from the ellipsoidal effects ( $\sim 5 \mathrm{mmag})$, we have split the full data set in 10 parts. For each part we used a single 5-th degree polynomial, and a number

13 See for example https://www. astro.keele.ac.uk/ jkt/debcat/debplots.htm 
Table 4. Comparison of our results for A- $083=\mathrm{RU}$ Cnc with literature. Please note the inversion of masses introduced by Imbert. Absolute values of $M$ and $R$ given in Popper (1990) and Imbert (2002) are estimates, and assume inclination $i=90^{\circ}$. Source of the $T_{\text {eff }}$ values in Çokluk et al. (2019) is unclear.

\begin{tabular}{lcccc}
\hline Parameter & Popper (1990) & Imbert (2002) (Tab. 2) & Çokluk et al. (2019) & This work \\
\hline \hline$P(\mathrm{~d})$ & $10.17289(-)$ & $10.172988(2)$ & $10.172918(3)$ & $10.1729311(55)$ \\
$K_{1}\left(\mathrm{~km} \mathrm{~s}^{-1}\right)$ & $70.4(1.2)$ & $67.50(71)$ & $68.19(8)$ & $70.61(9)$ \\
$K_{2}\left(\mathrm{~km} \mathrm{~s}^{-1}\right)$ & $69.9(1.2)$ & $70.46(64)$ & $70.69(8)$ & $67.2(3)$ \\
$i\left({ }^{\circ}\right)$ & $90(-)$ & $90(-)$ & $89.7(4)$ & $89(1)$ \\
$M_{1}\left(\mathrm{M}_{\odot}\right)$ & $1.46(7)$ & $1.42(4)$ & $1.437(46)$ & $1.349(23)$ \\
$M_{2}\left(\mathrm{M}_{\odot}\right)$ & $1.47(7)$ & $1.36(4)$ & $1.386(44)$ & $1.416(12)$ \\
$R_{1}\left(\mathrm{R}_{\odot}\right)$ & $1.9(-)$ & $1.89(2)$ & $2.392(69)$ & $2.19(4)$ \\
$R_{2}\left(\mathrm{R}_{\odot}\right)$ & $4.9(-)$ & $4.83(5)$ & $5.016(80)$ & $4.94(7)$ \\
$T_{\mathrm{eff}, 1}(\mathrm{~K})$ & - & - & $6860(285)$ & $6569(84)$ \\
$T_{\mathrm{eff}, 2}(\mathrm{~K})$ & - & - & $4800(200)$ & $4761(147)$ \\
\hline
\end{tabular}

$a$ In Table 3 of Imbert (2002) the masses are inverted, and in agreement with this work.

(between 5 and 7) of sine functions. For two parts form $\mathrm{C} 16$ we also added a variation in $l_{3}$ approximated by a 3 -rd degree polynomial.

As expected from such situation, the overall error budget of light-curve-based parameters was dominated by their $r m s$-es, which shows that we have properly taken the systematics into account. In case of the RVs, the activity and rotation again are the main sources of uncertainties for the cool secondary $\left(r m s \simeq 130 \mathrm{~m} \mathrm{~s}^{-1}\right.$ ), while the earlier type primary is more stable $\left(r m s \simeq 90 \mathrm{~m} \mathrm{~s}^{-1}\right.$ ). These values are, however, among the best in our sample, which allowed us to reach an excellent precision in masses $(\sim 0.2 \%$ for both primary and secondary), and also very good one in radii $(\sim 0.55-0.80 \%)$, which makes A-085 another example of a well-studied binary with an evolved, sub-giant component.

An interesting feature of A-085 is that it is part of a visual binary (ADS $7030 \mathrm{AB}, \rho \simeq 9$ arc-sec), and the whole system is a triple. The companion is separated far enough to obtain its spectra independently, therefore the information about the companion can be used to further constrain the age and metallicity of the whole system, or to verify results obtained for the eclipsing pair. Additionally, we also took advantage of the total part of the primary eclipse to obtain useful information (see Hełminiak et al. 2015, for another example). In total, the spectral analysis in ISPEC was performed on five spectra: disentangled primary, disentangled secondary, totality secondary (all from CHIRON), tertiary from HDS, and tertiary from CORALIE (shift-and-stack of three visits). Their SNR values were $68,46,40,103$, and 50, respectively. All five contributed to the final $[\mathrm{M} / \mathrm{H}]$, and the last two to constrain the age and verify the solution. The totality spectrum also allowed to verify if the TODCoR flux ratios and further renormalisation had produced reliable results. The averaged results for the tertiary's parameter are $T_{\text {eff }}=4994(161) \mathrm{K}$, and $\log (g)=3.39(28)$ dex, making it a sub-giant as well.

Our values of $M, R, T_{\mathrm{eff}}, l_{2} / l_{1}$ and $[\mathrm{M} / \mathrm{H}]$ are very well reproduced by a $\tau=2.40 \mathrm{Gyr},[\mathrm{Fe} / \mathrm{H}]=+0.15 \mathrm{dex}$ isochrone. The primary is at the main sequence, while the secondary evolved to a sub-giant, and is currently growing and cooling down, although not as quickly as the secondary in RU Cnc.

The isochrone reproduces the tertiary's atmospheric properties for $M \simeq 1.587 \mathrm{M}_{\odot}$, and a wide range of radii: $3.27<R<5.23 \mathrm{R}_{\odot}$. The Gaia Data Relase 2 (GDR2; Gaia Collaboration 2018) gives the effective temperature of ADS $7030 \mathrm{~B}$ at $4992_{-52}^{+84} \mathrm{~K}$, and the estimated radius is $4.78_{-0.15}^{+0.10} \mathrm{R}_{\odot}$. The latest LAMOST data release No. 5 (LDR5; Luo et al. 2019), gives a temperature 5012(15) K (average of two entries) and $\log (g)=3.359(25)$ dex, with metallicity slightly above solar $-0.085(15)$ dex. All those values agree reasonably well with our estimates, and the $2.40 \mathrm{Gyr}, 0.15 \mathrm{dex}$ isochrone.
Finally, the observed 2MASS $J-K$ colour index 0.56(2) mag is also very well reproduced by $\tau=2.40 \mathrm{Gyr},[\mathrm{Fe} / \mathrm{H}]=+0.15$ dex around $1.59 \mathrm{M}_{\odot}(0.53-0.59 \mathrm{mag})$. We therefore conclude that our solution for A-085 is consistent, evolutionary status of the whole triple is well established, and the visual companion B is likely a $\sim 1.59 \mathrm{M}_{\odot}$, $\sim 5000 \mathrm{~K}$ sub-giant.

\subsection{A-111 = FM Leo}

A-111 = FM Leo is the brightest target in our sample. It has been recognized by Maxted \& Hutcheon (2018) as a potentially very useful system for testing evolutionary models, since its brightness and low level of activity allow for very precise and accurate photometry and RVs. Unsurprisingly, it is listed in the DEBCat catalogue. Indeed, among our systems, A-111 has the lowest $r m s$ of both RV and light curves (Tab. 3). The $K 2$ data show relatively low-scale out-ofeclipse modulations, mainly ellipsoidal variations $(\sim 1 \mathrm{mmag})$ and long-time-scale trends (Fig. 3) Some obvious remnants from detrending also are noticeable, mainly discontinuities, or short-period oscillations. We split the SC light curve into five parts, and in the modelling we used a single 5-th degree polynomial and a single sine function, except for the first part where the sine was not used, and the fourth part, when two polys were used. The ISPEC analysis of disentagled spectra (SNR 117 and 95 for the primary and secondary, respectively) showed a small metal depletion, and very similar temperatures of the components.

We reached excellent precision in masses $(\sim 0.06 \%$ for both components), and also very good one in radii ( 0.55 and $0.8 \%)$, confirming the conclusions of Maxted \& Hutcheon (2018). This is thanks to the superb data, which include the richest set of highprecision RVs used for this object to date.

We found an excellent agreement of our results with a $[\mathrm{Fe} / \mathrm{H}]=0.05$ dex, $\tau=2.72 \mathrm{Gyr}$ isochrone. Both components are therefore on the main sequence. Comparison of our results with previously published works is shown in Table 5. The agreement is very good, except for $R_{2}$ from Sybilski et al. (2018), who only used the ASAS light curve, with no additional photometry. In particular we confirm the values of radii found by Maxted \& Hutcheon (2018) from $K 2$ long cadence light curve extracted with $\mathrm{k} 2 \mathrm{sFF}$. The advantage of using better precision RV measurements (Sybilski et al. 2018; Graczyk et al. 2021, and this work vs. previous studies) is clearly seen. The better precision reached by Graczyk et al. (2021) is most likely a result of their choice of the WD code to model the system, and to model the curve as a whole. This code, however, may be underestimating the systematic uncertainties, which could 
Table 5. Comparison of our results for A-111=FM Leo with literature. Values common for two works are given between their respective columns, and are originally derived in the earlier work.

\begin{tabular}{|c|c|c|c|c|c|}
\hline Parameter & Ratajczak et al. (2010) & Maxted \& Hutcheon (2018) & Sybilski et al. (2018) & Graczyk et al. (2021) & This work \\
\hline$P(\mathrm{~d})$ & $6.728606(6)$ & $6.728609(2)$ & $6.7286134(36)$ & $6.7286133(8)$ & $6.728606(2)$ \\
\hline$K_{1}\left(\mathrm{~km} \mathrm{~s}^{-1}\right)$ & \multicolumn{2}{|c|}{$76.62(27)$} & $75.992(29)$ & $76.017(10)$ & $76.033(19)$ \\
\hline$K_{2}\left(\mathrm{~km} \mathrm{~s}^{-1}\right)$ & \multicolumn{2}{|c|}{$78.46(28)$} & $78.653(36)$ & $78.654(15)$ & $78.646(22)$ \\
\hline$i\left(^{\circ}\right)$ & $87.98(6)$ & $87.96(1)$ & $89.07(63)$ & $87.941(23)$ & $87.939(28)$ \\
\hline$M_{1}\left(\mathrm{M}_{\odot}\right)$ & $1.318(7)$ & $1.32(1)$ & $1.3119(16)$ & $1.3144(5)$ & $1.3144(8)$ \\
\hline$M_{2}\left(\mathrm{M}_{\odot}\right)$ & $1.287(7)$ & $1.29(1)$ & $1.2675(14)$ & $1.2703(4)$ & $1.2707(7)$ \\
\hline$R_{1}\left(\mathrm{R}_{\odot}\right)$ & $1.648(43)$ & $1.634(5)$ & $1.76(8)$ & $1.625(2)$ & $1.627(9)$ \\
\hline$R_{2}\left(\mathrm{R}_{\odot}\right)$ & $1.511(49)$ & $1.498(6)$ & $1.22(11)$ & $1.508(3)$ & $1.498(12)$ \\
\hline$T_{\mathrm{eff}, 1}(\mathrm{~K})$ & $6316(240)$ & $6430(155)$ & - & $6397(56)$ & $6371(115)$ \\
\hline$T_{\text {eff }, 2}(\mathrm{~K})$ & $6190(211)$ & $6420(155)$ & - & $6386(56)$ & $6353(116)$ \\
\hline
\end{tabular}

be seen in the case of AI Phe in the thorough study of systematics and reliability in DEB modelling by Maxted et al. (2020).

Notably, Graczyk et al. (2021) reported detection of a Doppler beaming effect coming from only one of the components. Following the same approach, which uses the formalism presented in Placek (2019), one can conclude that both components of FM Leo should produce indistinguishable signals (dependent on $T_{\text {eff }}$ and $\log (g)$ ), that would cancel out. We suspect that the asymmetry found in their out-of-eclipse variations (long cadence data in these orbital phases) is caused by weak, cold spots.

Nevertheless, we conclude that FM Leo can be a real benchmark binary for testing stellar structure and evolution models.

\section{$5.6 \quad \mathrm{~A}-163$}

This is a system with the longest orbital period in our sample. The time span of the $K 2$ light curve is only $\sim 3$ times the orbital period, therefore a more precise determination of $P$ came from the RVs. The LC curve was fitted in Јктевор as a whole, but two different 4-th degree polynomials were used (for data taken before and after $J D=2456937.0$ ), together with five sine functions (Fig. 3). Because there are remnant oscillations seen in the residuals, we used the RS approach in Јктевор to assess realistic errors. Still, the resulting rms of the light curve fit is one of the lowest in our sample.

The RV data ( $r m s$ of 100 and $220 \mathrm{~m} \mathrm{~s}^{-1}$ for the primary and secondary, respectively) allow for excellent precision in mass determination, namely 0.28 and $0.18 \%$ for the primary and secondary, respectively. Coupled with high precision photometry, our data set led to a very good precision also in radii: $\sim 0.3 \%$ for both components. The ISPEC analysis of deconvolved spectra (SNR 127 and 38 for the primary and secondary, respectively) points towards subsolar metallicity stars of similar temperatures, but different $\log (g)$. As we did not assume synchronous rotation, we fitted for $v \sin (i)$ and obtained 14.1(7) and 5.7(2.8) $\mathrm{km} \mathrm{s}^{-1}$, for the primary and secondary, respectively. The primary is therefore rotating faster than in (pseudo-)synchronous configuration $\left(4.68 \mathrm{~km} \mathrm{~s}^{-1}\right)$. Its rotation may also be seen in the light curve - the sine with the largest amplitude was identified with $P=8.334 d$ (Tab. B2), which corresponds to $v \sin (i) \simeq 13.2 \mathrm{~km} \mathrm{~s}^{-1}$. The secondary also seems to rotate supersynchronously ( $2.66 \mathrm{~km} \mathrm{~s}^{-1}$ in tidal equilibrium), but the uncertainty is larger. According to JKTABSDIM results, synchronisation should occur at the age of $\sim 3.7 \mathrm{Gyr}$, which we can treat as an upper limit for the age of A-163.

In Table 6 we compare our results with previous works: by (1) Maxted \& Hutcheon (2018) who used only four publicly available FEROS spectra and calculated RVs with their own approach, (2)
Hełminiak et al. (2018), where many more spectra were used, but only the orbital solution has been updated with respect to the former, and (3) by Hoyman \& Çakırlı (2020) who used a mixture of literature and their own RV measurements, own light curve solution, and spectral analysis.

The improvement in precision of absolute parameters with respect to the earliest results is clear, coming mainly from the improved spectroscopic orbit. The precision in radii we obtained in this paper is therefore slightly better than in Hełminiak et al. (2018), although the errors in $r_{1,2}$ themselves (which are the same in Maxted \& Hutcheon 2018; Hełminiak et al. 2018) are a bit worse. This is possibly because Maxted \& Hutcheon (2018), did not take into account all possible sources of systematic errors, especially given the low number of eclipses in the $K 2$ data, or the influence of the third light, which we found small but not negligible. In any case, our independent analysis of a different RV set and with different approach to the light curve, led to high-quality results, consistent with the two previous studies.

Notably, the stellar parameters from the most recent study of Hoyman \& Çakırlı (2020) are not only of lower precision, but also show disagreement with other studies, mainly when comes to the primary component. This might have been caused by several factors, e.g. fixing $l_{2} / l_{1}$ to a value obtained from the $V$ band, instead of Kepler's, incorrect BJD calculated for the FEROS and HARPS spectra, higher measurement errors than in our approach to the same data, or obsolete version of the JкTEвор code used for light curve modeling. It is also not explained which $K 2$ data product was used, or was the light curve produced with a different software. We have also found some internal inconsistencies in Hoyman \& Çakırlı (2020). For example, taking the RVs as they are given in their Table A1 leads to $\mathrm{rms}$ of $1.4-1.7 \mathrm{~km} \mathrm{~s}^{-1}$, instead of 0.41-0.52 cited in Table 2. We could also not reproduce their results of $K_{1}$ and $K_{2}$, obtaining 45.3 and $54.1 \mathrm{~km} \mathrm{~s}^{-1}$, respectively ${ }^{14}$, which is more than $1 \sigma$ off the values given by them. Finally, applying their partial orbital, light curve, and spectral analysis results to JKTAвsDIM we could not reproduce the cited errors, nor the distance of 257(11) pc. The same apparent magnitudes (in $B, V, J, H$, and $K$, from Simbad), effective temperatures (from their spectral analysis) and fractional radii lead to $d \simeq 210 \mathrm{pc}$ (using surface brightness- $T_{\text {eff }}$ relations from Kervella et al., 2004). Finally, their ISPEC results suggest the secondary rotates faster $\left(16.3 \mathrm{~km} \mathrm{~s}^{-1}\right)$ than the primary $\left(14.7 \mathrm{~km} \mathrm{~s}^{-1}\right)$, while no such situation is observed in the width of the CCF peaks.

14 For this test, we used our v2FIT code and the measurements listed by Hoyman \& Çakırlı (2020). 
Table 6. Comparison of our results for A-163 with literature. Values common for two works are given between their respective columns, and are originally derived in the earlier work.

\begin{tabular}{|c|c|c|c|c|}
\hline Parameter & Maxted \& Hutcheon (2018) & Hełminiak et al. (2018) & Hoyman \& Çakırlı (2020) & This work \\
\hline$P(\mathrm{~d})$ & \multicolumn{2}{|c|}{$23.30962(5)$} & 23.309637(9) & $23.309595(35)$ \\
\hline$K_{1}\left(\mathrm{~km} \mathrm{~s}^{-1}\right)$ & $45.3(2.4)$ & $45.34(17)$ & $45.8(3)$ & $45.44(4)$ \\
\hline$K_{2}\left(\mathrm{~km} \mathrm{~s}^{-1}\right)$ & $57.8(2.6)$ & $56.35(21)^{a}$ & $55.1(7)$ & $56.56(7)$ \\
\hline$e^{2}$ & $0.027(2)$ & $0.0272(13)$ & $0.004(1)$ & $0.026(2)$ \\
\hline$\omega\left(^{\circ}\right)$ & $259.7(6)$ & $259(3)$ & 263.1(1.4) & $259(1)$ \\
\hline$i\left(^{\circ}\right)$ & \multicolumn{2}{|c|}{$88.65(1)$} & $88.6(17)^{b}$ & $88.668(14)$ \\
\hline$M_{1}\left(\mathrm{M}_{\odot}\right)$ & $1.48(16)$ & $1.407(12)$ & $1.35(5)$ & $1.421(4)$ \\
\hline$M_{2}\left(\mathrm{M}_{\odot}\right)$ & $1.16(13)$ & $1.132(9)$ & $1.12(6)$ & $1.141(2)$ \\
\hline$R_{1}\left(\mathrm{R}_{\odot}\right)$ & $2.18(7)$ & $2.154(7)$ & $1.63(5)$ & $2.157(6)$ \\
\hline$R_{2}\left(\mathrm{R}_{\odot}\right)$ & $1.22(4)$ & $1.209(6)$ & $1.18(5)$ & $1.224(4)$ \\
\hline$T_{\mathrm{eff}, 1}(\mathrm{~K})$ & \multicolumn{2}{|c|}{$6250(285)$} & $6550(150)$ & $6630(83)$ \\
\hline$T_{\mathrm{eff}, 2}(\mathrm{~K})$ & \multicolumn{2}{|c|}{$6150(285)$} & $6275(250)$ & $6586(380)$ \\
\hline
\end{tabular}

$a$ The value of $K_{2}$ in Table 1 of Hełminiak et al. (2018) is incorrectly given as $53.35 \mathrm{~km} \mathrm{~s}^{-1}$, which is a typographical error. The value given here is correct. Other parameters in Hełminiak et al. (2018), including those dependent on $K_{2}$ are given correctly.

$b$ Original notation from Table 2 in Hoyman \& Çakırlı (2020) with a probable typographic error.

For all these reasons we find the solution of Hoyman \& Çakırlı (2020) questionable, and suggest a cautious approach to their results for A-163 and other objects in their paper.

The MESA isochrone that best reproduces our results was found for the age of $2.40 \mathrm{Gyr}$ and metallicity of $-0.20 \mathrm{dex}$. The age is under the upper limit we obtained from the predicted synchronicity age, supporting the super-synchronous rotation of the primary. It also suggests a spin-orbit misalignment in this binary. The primary is somewhat evolved and is about to leave the main sequence. Interestingly, it lays at the edge of the $\gamma$ Dor theoretical instability strip, and its properties $\left(T_{\text {eff }}, \log (g), v_{\text {mic }}\right)$ fall into their observed distributions (Kahraman Aliçavuş et al. 2016). As was mentioned before, our model with 5 sines still does not cover all of the frequencies found in the data. One of the stronger and better separated remnants is a peak at $P=0.98 \mathrm{~d}$, which would be in agreement with the $v \sin (i)-\log \left(P_{\text {puls }}\right)$ relation shown in Kahraman Aliçavuş et al. (2016).

We therefore conclude that the primary of A-163 may be a $\gamma$ Dor-type star, but a more detailed analysis of its light curve is necessary, which was not the scope of this work. We would like to encourage the Community to pursue further investigation, by noticing that the TESS 30-min data are available from two sectors $(12,39)$, and the out-of-eclipse brightness variations are visible (with the $8.3 \mathrm{~d}$ peak being the dominant one). The measurement of the RV effect would also be welcome, as it could confirm the spin-orbit misalignment.

\section{$5.7 \quad$ A-171}

This system shows very little out-of-eclipse variations, except for ellipsoidal ( $\sim \mathrm{mmag}$ ) and imperfect detrending effects (Fig. 3). In particular, low level of activity allowed us to obtain relatively good rms of the light curve fit $(0.33 \mathrm{mag})$ and RV fit for both components $\left(0.54\right.$ and $0.26 \mathrm{~km} \mathrm{~s}^{-1}$, despite notable rotational broadening of $\sim 27 \mathrm{~km} \mathrm{~s}^{-1}$ ). The LC data set was fitted as a whole, with no additional polynomials or sine functions needed, but in this case it was necessary to free the reflection coefficients, and they were found to be $2.75(0.12) \times 10^{-3}$ and $2.58(0.11) \times 10^{-3}$ for the primary and secondary, respectively. Furthermore, the $K 2$ light curve covers $\sim 5.5$ orbital periods, so the analysis probably did not suffer much from systematics that might have originated from low number of data points and insufficient sampling.

We found that A-171 is composed of two very similar, but not identical F-type dwarfs. We reached a very good relative precision in masses and radii, namely $\sim 0.3 \%$ in $M$ and $0.4 \%$ in $R$. The spectroscopic analysis of the disentangled spectra (both of SNR 70) led to almost identical temperatures, which was expected from the nearly equal depths of eclipses. The metallicity was found to be solar within errors.

We note that both components of A-171 have similar mass to the primary of RU Cnc, but are significantly smaller and hotter. This, suggests that A-171 is younger than RU Cnc. The MESA isochrones nicely reproduce our results with the $\tau=2.29 \mathrm{Gyr},[\mathrm{Fe} / \mathrm{H}]=0.00 \mathrm{dex}$ isochrone. Both components are thus on the Main Sequence.

\section{$5.8 \quad$ A-231}

A-231 is another system with prominent spots that change rapidly, and thus the shape of its light curve varies from orbit to orbit (Fig. 3). However, in contrast to A-083 and A-085, the eclipses are partial, suggesting that the difference between two radii is not that significant as in the two aforementioned cases. The spot-induced brightness modulation is much weaker, not exceeding $5 \mathrm{mmag}$, yet larger than the ellipsoidal effect ( $\sim 1 \mathrm{mmag})$. The $K 2$ data from campaign $\mathrm{C} 12$ were split in 3 parts, and for each of them a single 5-th degree polynomial and five sine functions were used in the fitting. The data from campaign $\mathrm{C} 19$ were fitted with the aid of one 5-th degree poly and 2 sines, but they cover only a single orbital period. Remnant, short-time-scale brightness modulations are still visible in the residuals. Their origin is unclear to us, possibly a mixture of systematics coming from the detrending and real, intrinsic oscillations. As in the case of A-163, for error calculations in JKTEBOP we used the RS, which gives more reliable results in such cases. Nevertheless the overall quality of the fits was still very good, with the final $\mathrm{rms}$ of residuals of the fit at the level of $0.19 \mathrm{mmag}(0.11 \mathrm{mmag}$ outside of eclipses). The most important parameters $\left(r_{1,2}, J, l_{2} / l_{1}, i\right)$ obtained from various parts of the light curve turned out to be quite stable.

The activity did not significantly hamper the RV fit. With the rms of only 82 and $147 \mathrm{~m} \mathrm{~s}^{-1}$ for the primary and secondary, respectively, we were able to reach precision in masses at the level of $0.19-0.27 \%$. At the same time, the precision in radii was several 
times lower: 0.5 and $2.1 \%$ for the primary and secondary, respectively.

The ISPEC analysis of the disentangled spectra (SNR 167 and 75 for the primary and secondary, respectively) revealed that the system is most likely metal-depleted, and the components have quite similar temperatures - the primary had cooled significantly down during its MS evolution. Notably, this is the only case where $[\alpha / F e]$ was found different from 0.0 by more than $1 \sigma-0.10(7)$ dex.

Our estimates are very well reproduced by a $\tau=4.68 \mathrm{Gyr}$, $[\mathrm{Fe} / \mathrm{H}]=-0.25$ dex isochrone. A-231 is therefore the second oldest, and the most metal-depleted system in our sample. The metal depletion explains why the secondary is significantly larger than the Sun, despite having nearly the same mass and similar age.

\section{CONCLUSIONS}

We presented results of a combined $\mathrm{RV}+$ photometric + spectral analysis of eight detached eclipsing binaries from our spectroscopic survey, that were observed during the $K 2$ mission. Special attention was put to model the variable out-of-eclipse modulations, and properly account them for in the error budget.

The use of high-precision photometric data, together with good quality RV measurements, allowed us to obtain valuable results, especially absolute masses and radii, with fractional errors below $3 \%$, and very often below $1 \%$. The presented systems show interesting and rare properties, such as: low mass (secondary of A-045), multiplicity (A-045, A-085), possible pulsations and spin-orbit misalignment (A-163), later stages of evolution (A-085), including the first object crossing the Hertzsprung gap with $M$ and $R$ measured with high precision $(\mathrm{A}-083=\mathrm{RU} \mathrm{Cnc})$, or potential for obtaining results of extremely high precision of $0.1 \%$ and better $(\mathrm{A}-111=$ FM Leo), which is important for testing the latest models of stellar evolution (Valle et al. 2017). The Community is encouraged to pursue further, more detailed studies of the systems presented here.

We conclude that space-borne photometry opens possibility for extremely precise $<0.5 \%$ derivation of stellar radii, but only for systems that systems do not show prominent and unstable spots. Otherwise the precision is largely affected by their evolution in time which is likely disturbed by the detrending algorithms, and therefore has to be properly treated in error estimations. We claim that it is still possible to reach $\sim 0.1 \%$ level of radii uncertainty for highly-spotted DEBs, but light curve modelling of such data should include the dynamical character of the spots characteristics.

\section{ACKNOWLEDGEMENTS}

We would like to thank Dr. C. Gałan from the NCAC Warsaw for sharing with us his results of spectral analysis of FM Leo before the publication, and the anonymous Referees for the comments and corrections to the manuscript. This research made use of data collected at ESO under programmes 082.D-0499, 084.B-0029, 086.D-0078, 089.D-0097, 091.D-0145, 091.D-0414, 095.D-0026, 100.D-0273, 100.D-0339, 101.D-0697, and 102.D-0281 as well as through CNTAC proposals CN2011B-021, CN2012A-021, CN2012B-036, CN2013B-022, CN2014A-044, and CN2015A074. This research is based in part on data collected at Subaru Telescope, which is operated by the National Astronomical Observatory of Japan. We are honored and grateful for the opportunity of observing the Universe from Maunakea, which has the cultural, historical and natural significance in Hawaii. This work has made use of data from the European Space Agency (ESA) mission Gaia (https://www.cosmos.esa.int/gaia), processed by the Gaia Data Processing and Analysis Consortium (DPAC, https://www . cosmos. esa.int/web/gaia/dpac/consortium). Funding for the DPAC has been provided by national institutions, in particular the institutions participating in the Gaia Multilateral Agreement. K.G.H. is supported by the Polish National Science Center through grant no. 2016/21/B/ST9/01613. A.M., T.P., and M.K. are supported by the Polish National Science Center through grant no. 2017/27/B/ST9/02727. N.E. would like to thank the Gruber Foundation for its generous support to this research. A.J. and R.B. acknowledge support from ANID - Millennium Science Initiative - ICN12_009. A.J. acknowledges additional support from FONDECYT project 1210718. This work was supported by the Poznan University of Technology under the grant No. 0211/SBAD/0121, and by JSPS KAKENHI Grant Number $16 \mathrm{H} 01106$.

\section{DATA AVAILABILITY}

The data underlying this article are available in the article and in its online supplementary material.

\section{REFERENCES}

Armstrong D. J., et al. 2015, A\&A, 579, A19

Barros S. C. C., Demangeon O., Deleuil M., 2016, A\&A, 594, A100

Basri G., Shah R., 2020, ApJ, 901, 14

Bessell M. S., Castelli F., Plez B., 1998, A\&A, 333, 231

Blanco-Cuaresma S., Soubiran C., Heiter U., Jofré P., 2014, A\&A, 569, A111

Blanco-Cuaresma, S., et al. 2016, in 19th Cambridge Workshop on Cool Stars, Stellar Systems, and the Sun (CS19), 22

Brahm R., Jordán A., \& Espinoza N. 2017, PASP, 129, 034002

Cardelli J. A., Clayton G. C., Mathis J. S., 1989, ApJ, 345, 245

Ceraski W., 1911, AN, 187, 77

Choi J., Dotter A., Conroy C., Cantiello M., Paxton B., Johnson B. D., 2016, ApJ, 823, 102

Clausen J. V. et al., 2008, A\&A, 487, 1095

Code A. D., Bless R. C., Davis J., Brown R. H., 1976, ApJ, 203, 417

Çokluk K. A., Koçak D., Içli T., Karaköse S., Üstündağ S., Yakut K., 2019, MNRAS, 488, 4520

Conroy K. E., et al., 2020, ApJS, 250, 34

Coronado J., et al., 2015, MNRAS, 448, 1937

Cutri R. M., et al., 2003, yCat, II/246

Devor J., Charbonneau D., O’Donovan F. T., Mandushev G., Torres G., 2008, AJ, 135, 850

Dotter A., 2016, ApJS, 222, 8

Eggleton P. P., 1983, ApJ, 268, 368

Eggleton P. P., Yakut K., 2017, MNRAS, 468, 3533

Flower P. J., 1996, ApJ, 469, 335

Gaia Collaboration, 2016, A\&A 595, A1

Gaia Collaboration, 2018, A\&A, 616, A1

Gaia Collaboration, 2021, A\&A, 649, A1

Gillen E., et al., 2014, A\&A, 562, A50

Gillen E., et al., 2017, ApJ, 849, 11

Girardi L., et al., 2002, A\&A, 391, 195

Graczyk D., et al., 2021, A\&A, 649, A109

Gray R. O., Corbally C. J., 1994, AJ, 107, 742

Grevesse N., Asplund M., Sauval A. J., 2007, Space Sci. Rev., 130, 105

Gustafsson B., Edvardsson B., Eriksson K., Jørgensen U. G., Nordlund Å., Plez B., 2008, A\&A, 486, 951

Hełminiak K. G., Brahm R., Ratajczak M., Espinoza N., Jordán A., Konacki M., Rabus M., 2014, A\&A, 567, A64 
Hełminiak K. G., Jordán A., Espinoza N., Brahm R., Konacki M., 2018, RNAAS, 2, 226

Hełminiak K. G., Jordán A., Espinoza N., Brahm R., 2019c, Proc. of the International Astronomical Union, 15(S354), 300-304

Hełminiak K. G., et al., 2011, A\&A, 527, A14

Hełminiak K. G., et al., 2012, MNRAS, 425, 1245

Hełminiak K. G., et al., 2015, MNRAS, 448, 1945

Hełminiak K. G., et al., 2016, MNRAS, 461, 2896

Hełminiak K. G., et al., 2019a, A\&A, 622, A114

Hełminiak K. G., et al., 2019b, MNRAS, 484, 451

Hippke M., David T. J., Mulders G. D., Heller R., 2019, AJ, 158, 143

Huber D., et al., 2016, ApJS, 224, 2

Ho A. Y. Q., et al., 2017, ApJ, 836, 5

Howell S. B., et al., 2014, PASP, 126, 398

Hoyman B., Çakırlı Ö., 2020, MNRAS, 493, 2329

Ilijić S., Hensberge H., Pavlovski K., Freyhammer L. M., 2004, ASPC, 318 , 111

Imbert M., 2002, A\&A, 387, 850

Ioannidis P., Schmitt J. H. M. M., 2016, A\&A, 594, A41

Izumiura H., 1999, in: Proc. 4th East Asian Meeting on Astronomy, ed. P. S. Chen, Kunming, Yunnan Observatory, p. 77

Jordán A., et al., 2014, AJ, 148, 29

Kahraman Aliçavuş F., et al., 2016, MNRAS, 458, 2307

Kahraman Aliçavuş F., Soydugan E., Smalley B., Kubát J., 2017, MNRAS, 470,915

Kambe E., et al., 2013, PASJ, 65, 15

Kaufer A., et al. 1999, The Messenger, 95, 8

Kazarovets A. V., Samus N. N., Durlevich O. V., Frolov M. S., Antipin s. V., Kireeva N. N., Pastukhova E. N., 1999, IBVS, 4659, 1

Kervella P., Thévenin F., Di Folco E., Ségransan D., 2004, A\&A, 426, 297

Kiraga M., Stępien K., 2013, AcA, 63, 53

Klinglesmith D. A., Sobieski S., 1970, AJ, 75, 175

Konacki M., Muterspaugh M. W., Kulkarni S. R., Hełminiak, K. G., 2010, ApJ, 719, 1293

Kordopatis G., et al. 2013, AJ, 146, 134

Kurucz R. L., 1992, in Barbury B., Renzini A., eds, Proc. IAU Symp. 149, The Stellar Population of Galaxies, Kluwer Academic Publishers, Dordrecht, p. 225

Kunder A., et al. 2017, AJ, 153, 75

Lanza A. F., Rodonò M., Pagano I., 2004, A\&A, 425, 707

LaCourse D. M., et al. 2015, MNRAS, 452, 3561

Lastennet E., Valls-Gabaud D., 2002, A\&A, 396, 551

Lee C.-S., 2015, MNRAS, 453, 3474

Lucy L. B., 1967, ZA, 65, 89

Luger R., Agol E., Kruse E., Barnes R., Becker A., Foreman-Mackey D., Deming D., 2016, AJ, 152, 100

Luger R., Kruse E., Foreman-Mackey D., Agol E., Saunders N., 2018, AJ, 156,99

Luo A.-L., et al., 2019, yCat, V/164

Lurie J. C., et al., 2017, AJ, 154, 250

Marcadon F., et al., 2020, MNRAS, 499, 3019

Mason B. D., Wycoff G. L., Hartkopff W. I., Douglass G. G., Worley C. E., 2001, AJ, 122, 6

Maxted P. F. L., 2018, RNAAS, 2, 39

Maxted P. F. L., Hutcheon R. J., 2018, A\&A, 616, A38

Maxted P. F. L., et al., 2020, MNRAS, 498, 332

Moultaka J., Ilovaisky S. A., Prugniel P., Soubiran C., 2004, PASP 116, 693

Noguchi K., et al., 2002, PASJ, 54, 855

Paredes L. A., et al., 2021, AJ, 162, 167

Paxton B., Bildsten L., Dotter A., Herwig F., Lesaffre P., Timmes F., 2011, ApJS, 192, 3

Paxton B., et al., 2013, ApJS, 208, 4

Paxton B., et al., 2015, ApJS, 220, 15

Paxton B., et al., 2018, ApJS, 234, 34

Pepper J., et al., 2008, AJ, 135, 907

Petigura E. A., et al., 2015, ApJ, 811, 102

Placek B., 2019, Journal of Physics Conference Series, 1239, 012008

Pojmański G., 2002, AcA, 52, 397
Popper D. M., 1990, AJ, 100, 247

Popper D. M., Etzel P. B., 1981, AJ, 86, 102

Pourbaix D., et al. 2004, A\&A, 424, 727

Prša A., Zwitter T., 2005, ApJ, 628, 426

Ratajczak M., et al. 2010, MNRAS, 402, 2424

Ratajczak M., Hełminiak K. G., Konacki M., Jordán A., 2013, MNRAS, 433, 2357

Ratajczak M., et al. 2021, MNRAS, 500, 4972

Rucinski S. M., 1969, AcA, 19, 24

Schwab Ch., Spronck J., Tokovinin A., Szymkowiak A., Giguere M., Fisher D., 2012, Proc. SPIE, 8446, paper 8446-9

Shapley H., 1913, ApJ, 38, 158

Smith G. D., et al., 2021, arXiv:2109.00836

Somers G., Cao L., Pinsonneault M. H., 2020, ApJ, 891, 29

Southworth J., 2015, ASPC, 496, 164

Southworth J., Maxted P. F. L., Smalley B., 2004a, MNRAS, 351, 1277

Southworth J., Zucker S., Maxted P. F. L., Smalley B., 2004b, MNRAS, 355,986

Southworth J., Pavlovski K., Tamajo E., Smalley B., West R. G., Anderson D. R., 2011, MNRAS, 414, 3740

Stumpe M. C., et al., 2012, PASP, 124, 985

Sybilski P., Pawłaszek R. K., Sybilska A., Konacki M., Hełminiak K. G., Kozłowski S. K., Ratajczak M., 2018, MNRAS, 478, 1942

Ting Y.-S., Hawkins K., Rix H.-W., 2018, ApJL, 858, L7

Tokovinin A., et al., 2013, PASP, 125, 1336

Torres G., Andersen J., Giménez A., 2010, A\&ARv, 18, 67

Valle G., Dell'Omodarme M., Prada Moroni P. G., Degl'Innocenti S., 2017, A\&A, 600, A41

Vanderburg A., Johnson J. A., 2014, PASP, 126, 948

Voges W., et al. 1999, A\&A, 349, 389

Wilson R. E., Devinney E. J., 1971, ApJ, 166, 605

Wilson R. E., 2012, AJ, 144, 73

Windmiller G., Orosz J. A., Etzel P. B., 2010, ApJ, 712, 1003

Wraight K. T., White G. J., Bewsher D., Norton A. J., 2011, MNRAS, 416, 2477

Yıldı M., 2014, MNRAS, 437, 185

Zucker S., Mazeh T., 1994, ApJ, 420, 806

\section{APPENDIX A: INDIVIDUAL RV MEASUREMENTS}

In Table A1 we present individual RV measurements used in this work for orbital solutions. For both components of a given binary we show the measured $\mathrm{RV}$ values $v_{1,2}$, their errors $\epsilon_{1,2}$ (both in $\mathrm{km} \mathrm{s}^{-1}$ ), as well as exposure times in seconds and SNR at $\lambda \sim$ $5500 \AA$ A. The last column ("Inst.") codes the telescope, spectrograph, and observing mode used: $\mathrm{OH}=\mathrm{OAO}-188 / \mathrm{HIDES}, \mathrm{MF}=\mathrm{MPG}-$ $2.2 \mathrm{~m} / \mathrm{FEROS}, \mathrm{EC}=$ Euler/CORALIE, $\mathrm{CHf}$ and $\mathrm{CHs}=$ SMARTS $1.5 \mathrm{~m} / \mathrm{CHIRON}$ fiber and slicer, $\mathrm{EHc}$ and $\mathrm{EHg}=\mathrm{ESO}-3.6 \mathrm{~m} / \mathrm{HARPS}$ ECHE and EGGS, OE = OHP-1.93/ELODIE.

\section{APPENDIX B: K2 LIGHT CURVES}

In Table B1 and Figure B1 we present all the photometric data used in this study, as well as the model values (grey line in the figure) and residuals (in Table only). In the figure, each target and campaign is shown separately. If data from a single campaign were divided into parts, each part is drawn with a different colour. There may be systematic offsets in magnitude zero points, and differences between catalogue value of brightness in the Kepler band and maximum value on the plots may occur. This does not, however, affect the results of the LC analysis.

In Table B2 we list the values of polynomial coefficients and properties of the sine functions that were used to model the out-ofeclipse modulations. 
Table A1. All RVs used in this work for orbital solutions. Data for A-163 overlap with Hełminiak et al. (2018). The complete table is available as supplementary material.

\begin{tabular}{lcccrcc}
\hline \hline $\begin{array}{l}\text { BJD } \\
-2450000\end{array}$ & $\begin{array}{c}v_{1} \\
\left(\mathrm{~km} \mathrm{~s}^{-1}\right)\end{array}$ & $\begin{array}{c}v_{2} \\
\left(\mathrm{~km} \mathrm{~s}^{-1}\right)\end{array}$ & Target & $\begin{array}{r}t_{\text {exp }} \\
(\mathrm{s})\end{array}$ & SNR & Inst. \\
\hline 7021.117441 & $-81.04 \pm 0.89$ & - & A-045 & 900 & 62 & OH \\
7024.212351 & $-85.73 \pm 2.76$ & $114.31 \pm 4.19$ & A-045 & 2000 & 60 & OH \\
7059.095898 & $-87.85 \pm 1.02$ & $108.50 \pm 4.27$ & A-045 & 1800 & 36 & OH \\
$\ldots$ & & & & & & \\
6695.616152 & $131.66 \pm 1.63$ & $-90.29 \pm 0.72$ & A-060 & 900 & 43 & CHf \\
6707.544032 & $-17.95 \pm 0.83$ & $60.28 \pm 0.70$ & A-060 & 900 & 48 & CHf \\
6719.554731 & $-56.96 \pm 1.33$ & $101.15 \pm 0.90$ & A-060 & 900 & 35 & CHf \\
$\ldots$ & & & & & & \\
\hline
\end{tabular}

Table B1. $K 2$ photometric data used in this work, together with the resulting orbital phase and JктввоP model. The magnitude scale is arbitrary and may not coincide with the observed magnitude in the Kepler band. Data for A-111 = FM Leo (EPIC 201488365) were taken in short cadence. Only a portion is shown here, the full Table is available as supplementary material.

\begin{tabular}{lrrrrrc}
\hline BJD-245000 & Magnitude & Phase & Model & $(O-C)$ & EPIC ID & Camp. \\
\hline \hline 7820.581500 & 9.983082 & 0.86788862 & 9.984936 & -0.001854 & 247605441 & 13 \\
7820.601932 & 9.984303 & 0.88024685 & 9.985845 & -0.001543 & 247605441 & 13 \\
7820.622364 & 9.985404 & 0.89260495 & 9.986778 & -0.001374 & 247605441 & 13 \\
$\ldots$ & & & & & & \\
6770.433229 & 12.203283 & 0.09490711 & 12.203177 & 0.000106 & 202073040 & 0 \\
6770.453661 & 12.202893 & 0.10453924 & 12.202028 & 0.000865 & 202073040 & 0 \\
6770.474092 & 12.201474 & 0.11417089 & 12.200844 & 0.000630 & 202073040 & 0 \\
$\ldots$ & & & & & & \\
\hline
\end{tabular}
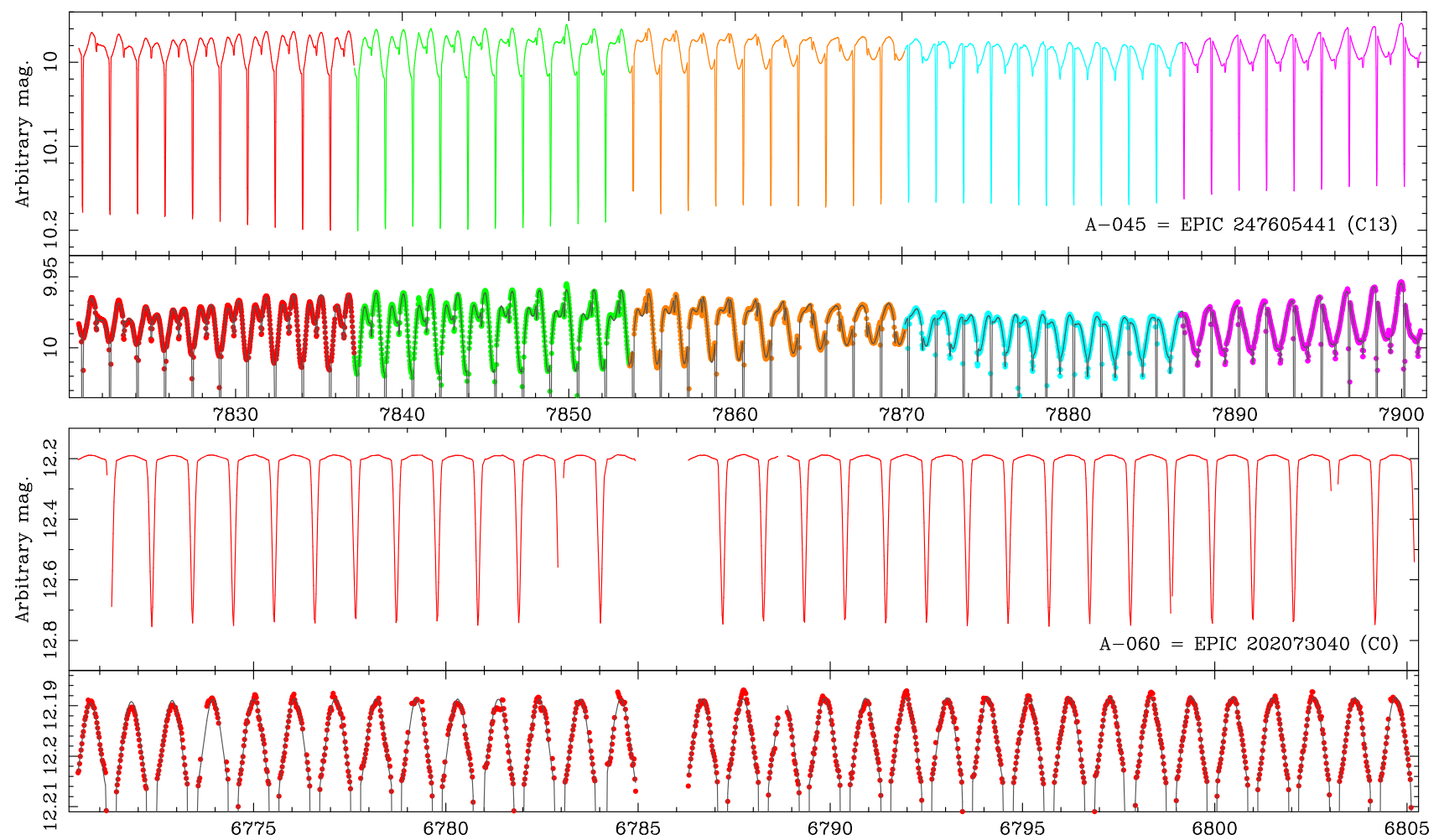

Figure B1. The $K 2$ light curves used in this work, shown as a function of time (BJD-2450000) and for each target and campaign separately. When analysis was done in parts, they are shown with different colours. The magnitude scale for A-085 is constant in all campaigns. Lower panels show zooms on the out-of-eclipse modulations, in order to better present the models (grey lines) and variations of the light curve shapes in time. The complete figure is available as supplementary material. 
Table B2. Information on polynomials and sines used in every individual JKTEBOP model. The polynomial is of the form $c_{0}+c_{1} x+c_{2} x^{2}+\ldots$ The values in "Beginning-End", "Pivot $i$ ", "Range $i$ ", ad $s T_{i}$ (sine's time base) are given as BJD-2450000. Sine's periods $\left(s P_{i}\right)$ and amplitudes $\left(s A_{i}\right)$ are given in days (d) and magnitudes (mag), respectively. The complete Table is available as supplementary material.

\begin{tabular}{|c|c|c|c|c|c|c|}
\hline \multicolumn{7}{|c|}{ Running name and EPIC ID } \\
\hline \multirow{8}{*}{$\begin{array}{l}\text { Campaign-Part } \\
\text { Beginning-End }\end{array}$} & $s T_{1}$ & $s P_{1}$ & $s A_{1}$ & $s T_{2}$ & $s P_{2}$ & $s A_{2}$ \\
\hline & $s T_{3}$ & $s P_{3}$ & $s A_{3}$ & $s T_{4}$ & $s P_{4}$ & $s A_{4}$ \\
\hline & $s T_{5}$ & $s P_{5}$ & $s A_{5}$ & $s T_{6}$ & $s P_{6}$ & $s A_{6}$ \\
\hline & $s T_{7}$ & $s P_{7}$ & $s A_{7}$ & & & \\
\hline & \multicolumn{2}{|c|}{ Range $_{1}$} & Pivot $_{1}$ & & & \\
\hline & \multirow{3}{*}{ Range } & $c_{11}$ & $c_{21}$ & $c_{31}$ & $c_{41}$ & $c_{51}$ \\
\hline & & & Pivot$_{2}$ & & & \\
\hline & & $c_{12}$ & $c_{22}$ & $c_{32}$ & $c_{42}$ & $c_{52}$ \\
\hline \multicolumn{7}{|c|}{$A-045=E P I C 247605441$} \\
\hline C13-1 & 7816.0969347285 & 1.6631766916 & 0.0128635335 & 7820.2936623448 & 0.8192959629 & 0.0094314870 \\
\hline \multirow{7}{*}{$7820.58-7837.12$} & 7821.0807661721 & 0.8353737272 & -0.0055340900 & 7821.1813222412 & 1.5723085304 & -0.0036088888 \\
\hline & 7820.7277959660 & 1.2677163863 & -0.0009682236 & 7821.6855947399 & 0.4077588724 & 0.0006512112 \\
\hline & 7820.8481968310 & 0.5391985203 & 0.0004684519 & & & \\
\hline & $7820.58-7$ & 37.12 & 7828.000 & & & \\
\hline & 0.0045582502 & -0.0005812301 & -0.0000828304 & 0.0000324034 & 0.0000009727 & 0.0000002941 \\
\hline & - & & - & & & \\
\hline & - & - & - & - & - & - \\
\hline \multirow{7}{*}{$\begin{array}{c}\text { C13-2 } \\
\text { 7837.12-7853.65 }\end{array}$} & 7839.3858457234 & 1.6425099802 & 0.0178860261 & 7840.2389735631 & 1.7936060255 & 0.0014213383 \\
\hline & 7840.3172866189 & 0.8193793759 & -0.0121861995 & 7842.4763761296 & 0.4066475451 & 0.0005301067 \\
\hline & 7840.3683741844 & 0.8571783149 & -0.0006999417 & 7842.3732345035 & 0.7934518107 & 0.0013810449 \\
\hline & 7842.0974614240 & 0.5463785489 & -0.0007092010 & & & \\
\hline & $7837.12-7$ & 53.65 & 7846.000 & & & \\
\hline & 0.0050098338 & -0.0001075007 & 0.0000359708 & 0.0000042225 & -0.0000002770 & -0.0000000447 \\
\hline & - & $\ldots$ & - & - & - & - \\
\hline \multirow{2}{*}{\multicolumn{7}{|c|}{$A-060=E P I C 202073040$}} \\
\hline & & & & & & \\
\hline $\mathrm{C} 0-1$ & - & - & - & - & - & - \\
\hline \multirow[t]{7}{*}{$6770.43-6805.19$} & - & - & - & - & - & - \\
\hline & - & - & - & - & - & - \\
\hline & - & - & - & & & \\
\hline & $6770.43-6$ & 05.19 & 6790.000 & & & \\
\hline & -0.0009548237 & -0.0000443111 & -0.0000006063 & 0.0000003812 & 0.0000000017 & -0.0000000011 \\
\hline & - & & - & & & \\
\hline & - & - & - & - & - & - \\
\hline$\ldots$ & & & & & & \\
\hline
\end{tabular}

\section{APPENDIX C: TESTING THE METHODOLOGY ON SYNTHETIC DATA}

The approach to model a non-trivial light curve of a DEB, by applying additional sines and polynomials, has been tested with synthetic data. We used the latest version (v2.3) of the PHysics Of Eclipsing BinariEs (PHOEBE; Prša \& Zwitter 2005; Conroy et al. 2020) software to generate eclipsing binary systems with spots that change in time. We based our synthetic binaries on A-045 and A-083 = RU Cnc, which have the lowest average precision in radii in our sample, providing the code with input stellar parameters (i.e. $M$, $R, T_{\text {eff }}, P, T_{0}$ ) from Table 3 . We also used exactly the same times of observations as in real $K 2$ curves for these two systems (from sectors 13 and 5, respectively). Various values of spot parameters (location, size, relative temperature) were used in each synthetic binary, so we could better revise their influence on the resulting parameters. In order to mimic the dynamical character of the synthetic light curve, we assumed non-synchronous rotation of components, and synchronicity parameters $F$ were varied from case to case. In this way we made sure that after every orbital cycle the location of spots changes (migration in longitude) and the shape of the light curve is different. When spots were included on different components we used different values of $F$ for each star. Finally, we added white noise, and (in some cases) additional long-term modulation with a 4-th degree polynomial. A total of six different synthetic light curves were made (three per binary), each showing a different pattern of spots that evolves in time.

They were analysed with ЈКТевоР in the same way as described in Section 4.2, i.e. each was divided into subsets, and for each subset we applied a number of sine functions and polynomials. For each subset, errors were estimated with a Monte-Carlo procedure (task 8). Results from each individual subset were then weight-averaged, and a combination of their rms and average error was used as the adopted uncertainty. The resulted values (with errors) of sum $\left(r_{1}+r_{2}\right)$ and ratio $(k)$ of fractional radii, inclination $(i)$, and fractional radii separately $\left(r_{1}\right.$ and $\left.r_{2}\right)$ are compared to their input values in Figure $\mathrm{C}$ 1. One can see that the results reproduce the input values with very good agreement, always within $1 \sigma$. It is worth to note, that while the shape of stars in PHOEBE is dictated by the Roche geometry, in ЈктевоР they are approximated by 2-axial ellipsoids. This did not affect the results, as expected for components with low oblateness.

This paper has been typeset from a $\mathrm{T}_{\mathrm{E}} \mathrm{X} / \mathrm{L} \mathrm{A} \mathrm{T}_{\mathrm{E}} \mathrm{X}$ file prepared by the author. 

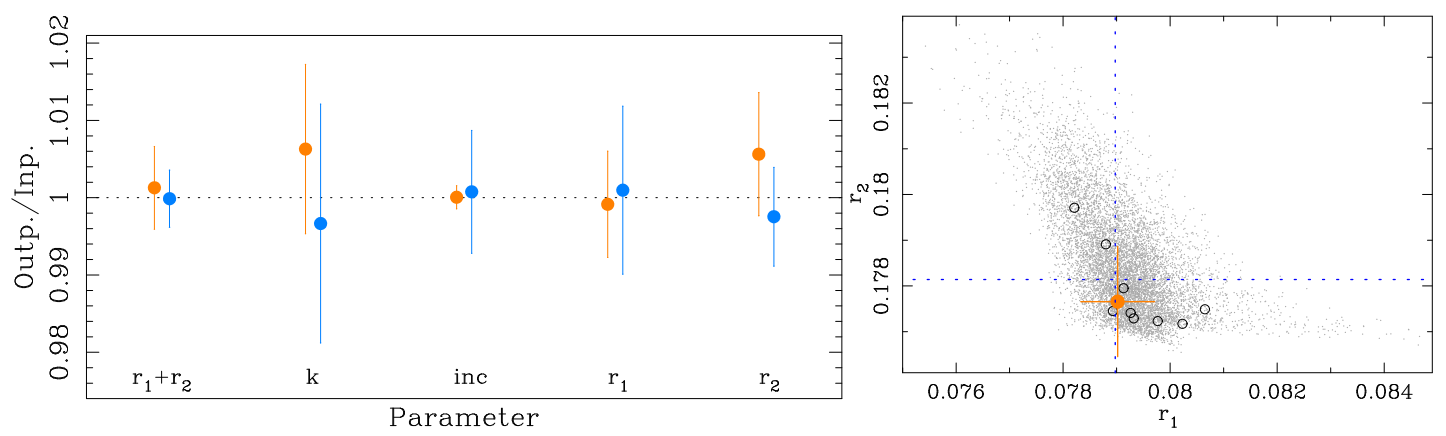

Figure C1. Left: Comparison of results of the Јктевор analysis of synthetic "spotted" light curves with the input values, for a sample of crucial parameters (fractional radii and inclination). The final results from all curves/subsets for the binary based on A-045 are plotted with orange symbols, and for the RU Cncbased system with blue. In all cases јктевор managed to accurately and precisely reproduce the input values. Right: Detailed results for the RU Cnc-based binary, on the $r_{1}-r_{2}$ plane. Grey dots show values from individual MC iterations ( 9000), black circles mark the best-fitting values from nine subsets, the orange symbol is the adopted result (with errors), and the blue dashed lines mark the input values. 